\title{
Minimal-Order Functional Observer-Based Residual Generators for Fault Detection and Isolation of Dynamical Systems
}

\author{
H. M. Tran and H. Trinh \\ School of Engineering, Deakin University, Geelong, VIC 3217, Australia \\ Correspondence should be addressed to H. Trinh; hieu.trinh@deakin.edu.au
}

Received 10 January 2016; Revised 22 March 2016; Accepted 5 April 2016

Academic Editor: Zehui Mao

Copyright ( $) 2016$ H. M. Tran and H. Trinh. This is an open access article distributed under the Creative Commons Attribution License, which permits unrestricted use, distribution, and reproduction in any medium, provided the original work is properly cited.

\begin{abstract}
This paper examines the design of minimal-order residual generators for the purpose of detecting and isolating actuator and/or component faults in dynamical systems. We first derive existence conditions and design residual generators using only first-order observers to detect and identify the faults. When the first-order functional observers do not exist, then based on a parametric approach to the solution of a generalized Sylvester matrix equation, we develop systematic procedures for designing residual generators utilizing minimal-order functional observers. Our design approach gives lower-order residual generators than existing results in the literature. The advantages for having such lower-order residual generators are obvious from the economical and practical points of view as cost saving and simplicity in implementation can be achieved, particularly when dealing with highorder complex systems. Numerical examples are given to illustrate the proposed fault detection and isolation schemes. In all of the numerical examples, we design minimum-order residual generators to effectively detect and isolate actuator and/or component faults in the system.
\end{abstract}

\section{Introduction}

Industrial systems have become more and more complicated and expensive, and thus the requirement of safety and reliability in system operations is paramount [1]. In fact, high complex modern engineering systems are vulnerable to unavoidable faults. Faults can happen due to an internal event, change in environmental conditions, error in the design of the system, sensor failure, actuator malfunctioning, or even human mistakes during operation. The unexpected faults disrupt the system operation, break down part of or the whole system, and can lead to fatal consequences. Fault detection and isolation (FDI) providing failure signals, therefore, have been considered as a vital aspect of system control research. This research issue has attracted extensive studies to safeguard equipment as well as amend the safety and reliability of modern system performance. Since the early 1970s, there have been a large number of fruitful results in FDI which can be found in, for example, [1-30] and the references therein.
In fault diagnosis area, there are two well-known model based fault diagnosis approaches. The first is the fault estimation based approach (see, e.g., [3-8]). By this approach, the systems are decoupled into fault-free and fault dependent parts. The faults can then be estimated via the observers of the fault-free part. The other is the residual-based approach which makes use of state observers to generate diagnostic signals or, in other words, residual generators. In [15], the authors provided a useful comparison between the two approaches. This study is interesting as it provides insightful knowledge and some shortcomings of each approach. The philosophy behind the residual-based approach is to estimate the system state vector based on the control inputs and the measured outputs. The residual generators are then constructed by a properly weighted output estimation error. The residuals are able to detect and identify the fault happening in the systems [9-14]. Obviously, the residual signals are expected to be close to zero in a fault-free condition and deviate from zero in the presence of a fault. For FDI purpose, a decision rule which is normally based on a threshold set is 
engaged to test for the likelihood of faults occurring in the systems.

Regarding the residual-based approach, the residual signals in most studies are generated via primarily employing full-order state observers or filter schemes which can be found in, for instance, [16-25]. However, the design and implementation of the residual generators using full-order state observers normally require high degrees of complexity and computational work, especially when dealing with highorder complex systems. As stated in [30], in certain applications such as fault diagnostic or control system design, an estimation of the entire state vector is not necessary. Meanwhile, there have been some FDI schemes based on reduced-order state observers (see, e.g., [26-29]). In these schemes, the residual generators are constructed based on the observers of the partial state vector. By that a significantly lower order of the residual generators, in comparison with the ones employing full-order state observers, can be achieved. With an attempt to simplify the design of the residual generators, this trend is identified as very important from the practical point of view (see [31-35]).

In this paper, by using minimal-order functional observers, we present a new FDI scheme to detect and identify $l$ unpredictable actuator and/or component fault signals entering from the inputs of dynamical systems. The systems considered in this paper have $n$ state variables, $p$ measured outputs, and $m$ control inputs. Our approach to FDI utilizes the advantages of functional observer schemes which can be found in [36-41]. Due to the fact that functional observers are employed instead of full-order state observers (or, for that matter, reduced-order Luenberger state observers) to generate the residual signals, substantial reduction in the complexity of the overall designed FDI schemes can now be consequently achieved. Particularly, we first present a simple solution approach where residual generators are designed using only first-order functional observers to detect and isolate faults in the systems. When first-order functional observers are not possible to design, then the residual generators are designed based on minimalorder functional observers. To minimize the observer order, we employ a parametric technique to the solutions of a generalized Sylvester matrix equation appearing in the existence conditions. Regarding the timely fault detection (FD), designing the residuals only involves the functional observers with the order as low as $[(n-p+1) / p]$, where $[x]$ denotes the smallest integer larger than $x$. This is a new finding and has not yet been reported in the literature. The result is significant since it is clear that, for $p>1$, the order of the residual generator is much lower than any of the existing results in the literature. Furthermore, for timely isolation of $l$ independent actuator and/or component faults in the system, we propose to construct a bank of $l$ residual generators, each with the order as low as $[(n-p+1) /(p-1)]$. Thus, it is clear that our proposed FD and FI schemes, taking advantage of functional observers, are identified as most beneficial for complex large-scale systems where, by default, $n$ and $p$ are large values.

The organization of this paper is as follows. In the next section (Section 2), we present the system description and preliminaries where we consider linear dynamical systems with $l$ unpredictable actuator and/or component fault signals entering from the system inputs. This section also introduces a novel residual generator that is constructed from reducedorder functional observers. Section 3 presents a detailed analysis on the design of minimal-order residual generators to trigger, in a timely manner, any fault that enters into the system. This follows by Section 4 where a bank of $l$ residual generators is designed to isolate $l$ likely faults. To achieve this, each residual generator is designed, using first-order or minimal-order functional observers, to be insensitive to one specific fault but sensitive to the remaining $(l-1)$ faults. Subsequently, a logic table can be drawn up from the $l$ residual generator outputs to detect and isolate the faults. Section 5 presents extensive numerical examples to highlight the attractive features of our proposed FDI schemes. Finally, Section 6 concludes the paper.

\section{System Description and Preliminaries}

In this paper, we consider dynamical systems with $p$ outputs and $m$ inputs and with unpredictable actuator fault signals $f(t) \in \mathbb{R}^{l}$ entering from the system inputs. In state space models, the systems are governed by the following equations:

$$
\begin{aligned}
& \dot{x}(t)=A x(t)+B u(t)+D f(t), \\
& y(t)=C x(t),
\end{aligned}
$$

where $x(t) \in \mathbb{R}^{n}, u(t) \in \mathbb{R}^{m}$, and $y(t) \in \mathbb{R}^{p}$ are the state, input, and output vectors, respectively. $A \in \mathbb{R}^{n \times n}, B \in \mathbb{R}^{n \times m}$, and $C \in \mathbb{R}^{p \times n}$ are known constant system matrices. $D \in$ $\mathbb{R}^{n \times l}$ is the fault identity matrix. We assume that $\operatorname{rank}(C)=$ $p$, without loss of generality, the fault identity matrix $D$ is also assumed to be a known full-column rank matrix, and the faults $f_{i}(t), i=1,2, \ldots, l$, are linearly independent. This assumption avoids vagueness which may appear when some faults occur simultaneously; as a result, the residual generators may not detect these faults due to the zero overall effect of these faults [26].

Let us consider the following reduced-order functional observer:

$$
\dot{\omega}(t)=N \omega(t)+G y(t)+H u(t),
$$

where $\omega(t) \in \mathbb{R}^{q}, 1 \leq q<n, N \in \mathbb{R}^{q \times q}, G \in \mathbb{R}^{q \times p}$, and $H \in \mathbb{R}^{q \times m}$ are observer parameters to be determined such that $\omega(t)$ is an asymptotic estimate of a linear function $L x(t)$ when no fault appears in the system, that is, $f(t)=0$, and $L \in \mathbb{R}^{q \times n}$ is a matrix to be determined for the purpose of FDI.

We now define a residual generator, $r(t)$, which is used to trigger the faults in the system:

$$
r(t)=T \omega(t)+F y(t)
$$

where $T \in \mathbb{R}^{1 \times q}$ and $F \in \mathbb{R}^{1 \times p}$ are residual parameters.

Figure 1 shows block-diagram implementation of the residual generator as defined in (2)-(3). By that, only known information of the inputs, $u(t)$, and the outputs, $y(t)$, of the system is utilized to generate the residual generator $r(t)$. 


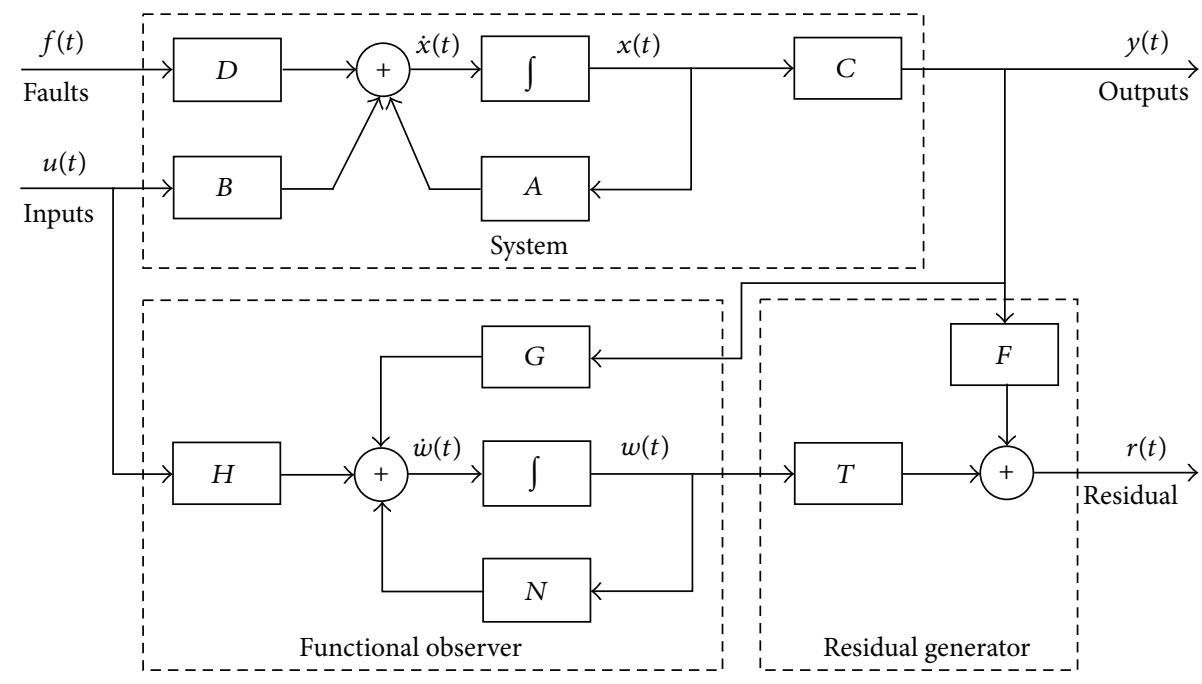

FIGURE 1: Block-diagram implementation of a residual generator.

Obviously, regarding the FD purpose, parameters $T, F$, $L, N, G$, and $H$ should be determined to meet the required functions of the residual generator such that

$$
\lim _{t \rightarrow \infty} r(t)= \begin{cases}0 & \text { if } f(t)=0 \\ c \text { or undefined } & \text { if } f(t) \neq 0,\end{cases}
$$

where $c \neq 0, f(t)=0$ implies a faultless condition, and $f(t) \neq$ 0 implies a faulty condition.

Error dynamics $e(t)$, which is defined to be the difference between the estimation $\omega(t)$ and the linear function $L x(t)$, is expressed as follows:

$$
e(t)=\omega(t)-L x(t) .
$$

By taking the derivative of the error vector (5) and then substituting $\dot{\omega}(t)$ from (2) and $\dot{x}(t)$ from (1), the error dynamics can be written as follows:

$$
\begin{aligned}
\dot{e}(t)= & N e(t)+(N L+G C-L A) x(t) \\
& +(H-L B) u(t)-L D f(t) .
\end{aligned}
$$

It is clear from (6) that, under the faultless condition, the error asymptotically converges to zero if the following conditions hold.

Proposition 1. Under faultless conditions, that is, $f(t)=$ $0, \omega(t)$ is an asymptotic estimate of $L x(t)$ (i.e., $e(t) \rightarrow 0$ asymptotically) for any initial conditions $x(0)$ and $\omega(0)$ if

$$
N \text { is Hurwitz, }
$$

$$
\begin{gathered}
N L+G C-L A=0, \\
H-L B=0 .
\end{gathered}
$$

Proof. When no fault occurs in the system, if conditions (8) and (9) are satisfied then (6) is reduced to $\dot{e}(t)=N e(t)$. Thus, if $N$ is Hurwitz then $e(t) \rightarrow 0$ asymptotically. Note also that if all the eigenvalues of $N$ can be arbitrarily assigned, then $e(t) \rightarrow 0$ with any prescribed rate. This completes the proof of Proposition 1.

Definition 2. The functional observer (2) is an Asymptotic Estimator if $\omega(t)$ is an asymptotic estimate of $L x(t)$ when $f(t)=0$.

Since Proposition 1 is satisfied and based on Definition 2, the error dynamics of an asymptotic observer takes the following form:

$$
\dot{e}(t)=N e(t)-L D f(t) .
$$

Substituting (5) into (3), the residual generator $r(t)$ can be obtained as follows:

$$
r(t)=T e(t)+(T L+F C) x(t) .
$$

According to (4) and (11), the residual generator can satisfy its proposed functions if the following conditions hold.

Proposition 3. An Asymptotic Estimator of form (2) can satisfy $r(t)$ according to (4) for any $x(0)$ and $\omega(0)$ if

$$
\begin{array}{r}
T L+F C=0, \\
L D \neq 0 .
\end{array}
$$

Proof. If $f(t)=0$ and also (12) and (13) are satisfied then according to (10) we have $e(t) \rightarrow 0$ asymptotically and according to (11) we have $r(t) \rightarrow 0$ asymptotically. When a fault appeared in the system, that is, $f(t) \neq 0$, and condition (13) holds, based on (10) we have $e(t) \rightarrow 0$. Accordingly, the residual signals (11) deviate from zero, which indicates the fault happened in the system. Thus, the required functions of the residual generator as stated in (4) are satisfied. This completes the proof of Proposition 3. 
Now, the design of the functional observer (2) and the residual generator (3) reduces to determining the unknown matrices $L, N, G, H, T$, and $F$ such that conditions (7)-(9), (12), and (13) are satisfied.

\section{Fault Detection Scheme}

In Section 2, we showed that the functional observer (2) and the residual generator (3) can detect faults in the system if all the unknown parameters satisfying the conditions stated in Propositions 1 and 3 are found. Observe that if $L$ is known, then from (9), $H=L B$, and condition (13), that is, $L D \neq 0$, can be easily verified. In this section, we propose a systematic procedure for solving coupled matrix equations (8) and (12) with the requirement that matrix $N$ is Hurwitz. Note that, here, not only do we require that matrix $N$ be Hurwitz, but also we desire its eigenvalues to be placed at some prespecified locations in the $s$-plane in order to achieve timely detection of faults.

Let us first simplify matrix equations (8) and (12) by a partition technique defined in [38]. Regarding this, an invertible matrix $P \in \mathbb{R}^{n \times n}$ is introduced:

$$
P=\left[\begin{array}{ll}
C^{+} & C^{\perp}
\end{array}\right]
$$

where $C^{+} \in \mathbb{R}^{n \times p}$ denotes the Moore-Penrose inverse of $C$, that is, $C C^{+}=I_{p}$, and $C^{\perp} \in \mathbb{R}^{n \times(n-p)}$ denotes an orthogonal basis for the null-space of $C$, that is, $C C^{\perp}=0$. Now the following partitions are defined:

$$
\begin{aligned}
C P & =\left[\begin{array}{ll}
I_{p} & 0
\end{array}\right], \\
L P & =\left[\begin{array}{ll}
L_{1} & L_{2}
\end{array}\right], \\
P^{-1} A P & =\left[\begin{array}{ll}
A_{11} & A_{12} \\
A_{21} & A_{22}
\end{array}\right],
\end{aligned}
$$

where submatrices $L_{1} \in \mathbb{R}^{q \times p}, L_{2} \in \mathbb{R}^{q \times(n-p)}, A_{11} \in \mathbb{R}^{p \times p}$, $A_{12} \in \mathbb{R}^{p \times(n-p)}, A_{21} \in \mathbb{R}^{(n-p) \times p}$, and $A_{22} \in \mathbb{R}^{(n-p) \times(n-p)}$.

Postmultiplying both sides of (8) and (12) by the matrix $P$, we obtain

$$
\begin{array}{r}
N L P+G C P-L P P^{-1} A P=0, \\
T L P+F C P=0 .
\end{array}
$$

By substituting (15)-(17) into (18), we achieve the following equations:

$$
\begin{aligned}
G & =L_{1} A_{11}+L_{2} A_{21}-N L_{1}, \\
F & =-T L_{1}, \\
N L_{2}-L_{2} A_{22}-L_{1} A_{12} & =0 \\
T L_{2} & =0 .
\end{aligned}
$$

It is clear from (19) and (20) that matrices $G$ and $F$ can be computed once $N, L_{1}, L_{2}$, and $T$ are found to satisfy (21) and (22).
In the following, we discuss the solutions to coupled matrix equations (21) and (22). Observe that the solution to (21) depends on the characteristic of matrix $A_{12} \in \mathbb{R}^{p \times(n-p)}$. Hence, we classify $A_{12}$ into two cases, namely, Case 1 where $A_{12}$ is a column matrix and Case 2 where $A_{12}$ is a row matrix.

Case 1 (first-order residual generators). In this case, we consider that $p>n / 2$; accordingly $A_{12}$ is a column matrix. For this, we only need to design a first-order residual generator to detect faults in the system. Regarding this, the design procedure is detailed as follows.

Theorem 4. A first-order functional observer (2) always exists for system (1) when $A_{12}$ is a column matrix; that is, $p>n / 2$. Furthermore, a residual generator (3) can be constructed to detect faults in the system if condition (13) is satisfied.

Proof. To design a first-order functional observer, that is, $q=$ $1, N \in \mathbb{R}^{1 \times 1}$ can be chosen to be any negative scalar. Thus, let

$$
N=s, \quad s<0
$$

By letting $L_{2}=0,(21)$ is reduced to the following:

$$
L_{1} A_{12}=0 \text {. }
$$

Since $A_{12}$ is a column matrix, a solution to (24) where $L_{1} \neq 0$ always exists. Let $\mathcal{N}(X)$ be a matrix of row basis vectors for the row-null-space of $X$; that is, $\mathcal{N}(X) X=0$. Therefore, the solution for $L_{1}$ in (24) can be achieved by first computing $\widehat{L}_{1}$ according to

$$
\widehat{L}_{1}=\mathscr{N}\left(A_{12}\right)
$$

and then $L_{1}$ can be selected as any row of $\widehat{L}_{1}$.

Matrix $T \neq 0$ in (22) can be arbitrarily chosen to be any nonzero scalar, say, $\alpha$, since $L_{2}=0$. Finally, if condition (13) is satisfied, a residual generator using a first-order functional observer exists to detect faults in the system. Matrices $H, G$, and $F$ can then be easily obtained from (9), (19), and (20), respectively, to complete the design of the residual generator. This completes the proof of Theorem 4.

It is worth pointing out that, in the derivation of a firstorder residual generator, we do not impose the requirement that the matrix pair $(A, C)$ is observable.

Case 2 (minimal-order residual generators). In this case, we consider that $1<p \leq n / 2$; accordingly $A_{12}$ is a row matrix. Matrix $A_{12}$ is considered to be full rank; that is, $\operatorname{rank}\left(A_{12}\right)=p$. For this, we design a minimal-order residual generator for FD by presenting a solution to coupled matrix equations (21) and (22) via a parametric technique. Here, $N$ has preassigned distinct eigenvalues satisfying condition (7). For completeness, let us first present a parametric solution [42] to generalized Sylvester matrix equation (21). For the solution of (21), we require that the pair $\left(A_{12}, A_{22}\right)$ be observable. This implies that the pair $(C, A)$ is observable [38]. 
Let $N \in \mathbb{R}^{q \times q}$ with $q$ distinct eigenvalues be defined as follows:

$$
N=Q^{-1} \Lambda Q
$$

where $Q \in \mathbb{R}^{q \times q}$ is any freely chosen invertible matrix and $\Lambda=\operatorname{diag}\left(s_{1}, s_{2}, \ldots, s_{q}\right), s_{i} \neq s_{j}$ for $i \neq j$ and $\operatorname{Re}\left\{s_{i}\right\}<0$ for all $i=1,2, \ldots, q$. With $N$ as defined in (26), $L_{1}$ and $L_{2}$ satisfying (21) are given in the following parametric forms [42]:

$$
\begin{aligned}
& L_{1}=Q\left[\begin{array}{llll}
U\left(s_{1}\right) b_{1} & U\left(s_{2}\right) b_{2} & \cdots & U\left(s_{q}\right) b_{q}
\end{array}\right]^{\top}, \\
& L_{2}=Q\left[\begin{array}{llll}
Z\left(s_{1}\right) b_{1} & Z\left(s_{2}\right) b_{2} & \cdots & Z\left(s_{q}\right) b_{q}
\end{array}\right]^{\top},
\end{aligned}
$$

where $b_{i} \in \mathbb{C}^{p}(i=1,2, \ldots, q)$ are free vectors satisfying $b_{i}=$ $\bar{b}_{j}$ if $s_{i}=\bar{s}_{j} . \bar{s}_{j}$ denotes the complex conjugate of $s_{i} . U(s) \in$ $\mathbb{R}^{p \times p}$ and $Z(s) \in \mathbb{R}^{(n-p) \times p}$ are coprime polynomial matrices satisfying the following coprime factorization:

$$
\left(s I_{n-p}-A_{22}^{\top}\right)^{-1} A_{12}^{\top}=Z(s) U^{-1}(s) \text {. }
$$

The reader can refer to [42] for a numerically reliable algorithm to compute $Z(s)$ and $U(s)$. Also, as suggested in [38], $U(s)$ and $Z(s)$ can be conveniently computed according to the following equations:

$$
\begin{aligned}
& U(s)=\operatorname{det}\left(s I_{n-p}-A_{22}\right) I_{p}, \\
& Z(s)=\operatorname{adj}\left(s I_{n-p}-A_{22}^{\top}\right) A_{12}^{\top},
\end{aligned}
$$

where $\operatorname{det}(\cdot)$ and $\operatorname{adj}(\cdot)$ denote the determinant and the adjugate matrix of matrix $(\cdot)$, respectively. For any given $A_{22}$, the characteristic polynomial can be obtained:

$$
\begin{aligned}
a(s) & \triangleq \operatorname{det}\left(s I_{n-p}-A_{22}\right) \\
& =s^{n-p}+a_{1} s^{n-p-1}+a_{2} s^{n-p-2}+\cdots+a_{n-p},
\end{aligned}
$$

where the coefficients $a_{i}, i=1,2, \ldots,(n-p)$, are real constants. The adjugate matrix $\operatorname{adj}(\cdot)$ is then obtained as follows:

$$
\begin{aligned}
\operatorname{adj}\left(s I_{n-p}-A_{22}^{\top}\right)= & \Upsilon_{1} s^{n-p-1}+\Upsilon_{2} s^{n-p-2}+\Upsilon_{3} s^{n-p-3} \\
& +\cdots+\Upsilon_{n-p},
\end{aligned}
$$

where $\Upsilon_{i}, i=1,2, \ldots, n-p$, are computed by using the coefficients of $a(s)$ and matrix $A_{22}$, where

$$
\begin{gathered}
\Upsilon_{1}=I_{n-p}, \\
\Upsilon_{2}=\Upsilon_{1} A_{22}^{\top}+a_{1} I_{n-p}, \\
\Upsilon_{3}=\Upsilon_{2} A_{22}^{\top}+a_{2} I_{n-p}, \\
\vdots \\
\Upsilon_{n-p}=\Upsilon_{n-p-1} A_{22}^{\top}+a_{n-p-1} I_{n-p} .
\end{gathered}
$$

Note that if the preassigned eigenvalues of $N$ are complex then the resulting matrices $\Lambda, L_{1}$, and $L_{2}$ are also complex. As reported in [14], we can obtain real matrices $\Lambda, L_{1}$, and $L_{2}$ by applying the following simple computation. Here, without loss of generality, we assume that $s_{1}$ and $s_{2}, s_{2}=\bar{s}_{1}=\alpha+\beta j$, are a pair of the eigenvalues containing complex values, and all other eigenvalues of $N$ are real and distinct. The real matrices $\Lambda, L_{1}$, and $L_{2}$ can be obtained as follows:

$$
\begin{aligned}
& \Lambda=\text { block- } \operatorname{diag}\left(\Lambda_{1}, s_{3}, \ldots, s_{q}\right), \\
& \Lambda_{1}=\left[\begin{array}{cc}
\alpha & \beta \\
-\beta & \alpha
\end{array}\right], \\
& L_{1} \\
& =Q\left[\begin{array}{llll}
\operatorname{Re}\left\{U\left(s_{1}\right) b_{1}\right\} & \operatorname{Im}\left\{U\left(s_{1}\right) b_{1}\right\} & \cdots & U\left(s_{q}\right) b_{q}
\end{array}\right]^{\top}, \\
& L_{2} \\
& =Q\left[\begin{array}{llll}
\operatorname{Re}\left\{Z\left(s_{1}\right) b_{1}\right\} & \operatorname{Im}\left\{Z\left(s_{1}\right) b_{1}\right\} & \cdots & Z\left(s_{q}\right) b_{q}
\end{array}\right]^{\top},
\end{aligned}
$$

where $\operatorname{Re}\{\cdot\}$ and $\operatorname{Im}\{\cdot\}$ denote the real and the imaginary parts of $\{\cdot\}$, respectively.

Now, by incorporating $L_{2}$ as defined in (28) into (22), we can solve for $T$. Let us now present the result for Case 2 by the following theorem.

Theorem 5. With $1<p \leq n / 2$ and $\operatorname{rank}\left(A_{12}\right)=p$, a residual generator (3) with an order as low as $q=[(n-p+1) / p]$ can be constructed to detect faults in the system if condition (13) is satisfied.

Proof. First, let us express TQ as follows:

$$
T Q=\left[\begin{array}{llll}
t_{1} & t_{2} & \cdots & t_{q}
\end{array}\right]
$$

where $t_{i} \neq 0, i=1,2, \ldots, q$, are arbitrarily real numbers.

Once $t_{i}$ are scalars, by substituting (28) and (36) into the transpose of (22) and after some rearrangement the following equation is obtained:

$$
\left[\begin{array}{llll}
Z\left(s_{1}\right) t_{1} & Z\left(s_{2}\right) t_{2} & \cdots & Z\left(s_{q}\right) t_{q}
\end{array}\right]\left[\begin{array}{c}
b_{1} \\
b_{2} \\
\vdots \\
b_{q}
\end{array}\right]=0 .
$$

Now, (37) can be expressed as follows:

$$
\mathscr{Z b}=0 \text {, }
$$

where $\mathscr{Z} \in \mathbb{R}^{(n-p) \times q p}$ and $b \in \mathbb{R}^{q p}$ and

$$
\begin{aligned}
\mathscr{Z} & =\left[\begin{array}{lllll}
Z\left(s_{1}\right) t_{1} & Z\left(s_{2}\right) t_{2} & \cdots & Z\left(s_{q}\right) t_{q}
\end{array}\right], \\
b & =\left[\begin{array}{llll}
b_{1}^{\top} & b_{2}^{\top} & \cdots & b_{q}^{\top}
\end{array}\right]^{\top} .
\end{aligned}
$$


Similar to the solution to $(24)$, let $\mathcal{N}(\mathscr{Z})$ be defined such that $\mathscr{Z} \mathscr{N}(\mathscr{Z})=0$; then a solution to (38) always exists for $b \neq 0$ if $\mathscr{N}(\mathscr{Z}) \neq 0$. Accordingly, if $\mathscr{Z}$ is a row matrix, that is, the number of columns is greater than the number of rows, the solution to $b \neq 0$ always exists. That implies the following result:

$$
q \geq\left[\frac{n-p+1}{p}\right]
$$

and then $b \neq 0$ exists and can be taken to be any column of $\mathcal{N}(\mathscr{Z})$.

As a result, with $q$ prescribed eigenvalues for $N$ (i.e., $s_{i}, i=$ $1,2, \ldots, q$, are given) and for any arbitrary invertible matrix Q, matrices $N, L_{1}$, and $L_{2}$ are computed based on (26), (27), and (28), respectively. Matrix $L$ is then calculated by (16) and condition (13) can be verified. If (13) is satisfied, the residual generator $r(t)$ can detect faults in the system. Finally, $H, G, F$, and $T$ are obtained from (9), (19), (20), and (36), respectively, to complete the design of the observer $\omega(t)$ and the residual $r(t)$. This completes the proof of Theorem 5 .

Remark 6. The observer order $q$ can be assigned to satisfy condition (40), where a solution to (38) always exists for $b \neq 0$. Thus, for the purpose of designing minimal-order observers, we only need to choose $q=[(n-p+1) / p]$.

Remark 7. External disturbances or the uncertainties are unavoidable during the operation of the systems. If we take disturbances in the system where the disturbance matrix is defined as $E \in \mathbb{R}^{n \times r}$ and the disturbance vector is $d(t) \in \mathbb{R}^{r}$, system (1) now can be governed by the following equations:

$$
\begin{aligned}
& \dot{x}(t)=A x(t)+B u(t)+D f(t)+E d(t), \\
& y(t)=C x(t) .
\end{aligned}
$$

Based on the disturbance decoupling approach [7], the influence of the unknown disturbance can be eliminated. Using this approach, the conditions in Proposition 1 of this paper are as follows:

$$
\begin{gathered}
N \text { is Hurwitz, } \\
N L+G C-L A=0, \\
H-L B=0, \\
L E=0 .
\end{gathered}
$$

Since the condition $L E=0$ is added, the solutions to the observer parameters will be more strict. Nevertheless, based on the solution approach in this paper, it is possible to design minimal-order observer-based residual generators to detect faults in the systems with unknown disturbances. In this case, the residual functions are also defined as (4) which means the threshold can be set as zero. In addition, it is also possible to extend the result to include nonlinearities in the systems. This topic deserves further research and will be a subject for future research. Another issue that deserves further research is related to the robust threshold selection. How to solve the robust threshold selection problem under uncertainties is an important research question and the reader is referred to the most recent and interesting research [43].

Remark 8 . We conclude this section by considering the case where $1<p \leq n / 2$ and $\operatorname{rank}\left(A_{12}\right)<p$. Note that, for this case, a first-order residual generator can be designed since a solution to (24) where $L_{1} \neq 0$ exists due to the fact that $\operatorname{rank}\left(A_{12}\right)<p$. Indeed, there exists $\mathscr{N}\left(A_{12}\right) \neq 0$ such that $\mathcal{N}\left(A_{12}\right) A_{12}=0$. Accordingly, $L_{1} \neq 0$ is obtained and the rest of the observer and residual generator parameters can be derived in the same way as reported in Case 1. A numerical example can be easily constructed to demonstrate this case. However, due to space limiting reason, a numerical example is not given in this paper.

\section{Fault Isolation Scheme}

In Section 3, we discussed the design of minimal-order residual generators for FD in the systems. However, identifying the faults is also considered as an important task in system control since it provides the fault positions [15]. The information about the fault positions is then sent to the decision-maker for further actions dealing with the faults. In this regard, we present a systematic procedure to construct a bank of minimal-order residual generators which is used to isolate the faults. Hence, our proposed FI scheme significantly reduces the complexity in the implementation. The scheme is thus more suited to deal with complex high-order systems. Before showing the procedure, let us mention a logic for the fault isolation from the following [44].

We consider, in this paper, that there may be $l$ likely faults occurring in the system. Accordingly, for the purpose of FI, we propose to design a bank of $l$ residual generators, in which each residual generator is expected to be insensitive to one specific fault but sensitive to the rest of the remaining faults. Thus, a $k$ th residual generator, $r_{k}(t)$, is designed to be insensitive to the $k$ th fault, that is, $f_{k}(t)$, and sensitive to the rest of the remaining faults. By that, when there is no fault in the system, the outputs of the residual generators remain as zero. However, when a fault, say, $f_{k}(t)$, has occurred, the output of the $k$ th residual generator remains as zero; meanwhile, the outputs of the other $(l-1)$ residual generators are not zero but turn to $c_{k} \neq 0$, where $c_{k}$ is as defined in (52). Consequently, by measuring the output values of the $l$ residual generators or using a set of residual thresholds, when a fault appears, we can identify the position of the fault.

For simpler explaining of the logic, let us take an example where there are four possible faults, that is, $f_{k}(t), k=1,2,3,4$, in the system. Accordingly, a bank of four minimal-order residual generators, $r_{k}(t), k=1,2,3,4$, is designed to isolate the faults. Let us use a logic " 1 " to indicate that the output of a residual generator is bigger than a threshold value, that is, $c_{k} \neq 0$, and a logic " 0 " to indicate that the output of a residual generator remains as zero (or less than a threshold value). Table 1 shows the logic for the fault isolation. In the table, for instance, when a fault $f_{1}(t)$ has occurred, residual $r_{1}(t)$ is insensitive to $f_{1}(t)$; hence it appears as " 0 ." Meanwhile, other residuals can trigger $f_{1}(t)$ so they are expressed as "1." Therefore, we can identify that fault $f_{1}(t)$ has occurred. 
TABLE 1: Fault isolation logic table.

\begin{tabular}{lcccc}
\hline & $r_{1}(t)$ & $r_{2}(t)$ & $r_{3}(t)$ & $r_{4}(t)$ \\
\hline No fault & 0 & 0 & 0 & 0 \\
$f_{1}(t)$ & 0 & 1 & 1 & 1 \\
$f_{2}(t)$ & 1 & 0 & 1 & 1 \\
$f_{3}(t)$ & 1 & 1 & 0 & 1 \\
$f_{4}(t)$ & 1 & 1 & 1 & 0 \\
\hline
\end{tabular}

However, it is noted that this logic is only correct when the faults happen independently and also not simultaneously. In Section 2, we have made an assumption that avoids such a situation and hence the logic expressed in Table 1 can thus be used to isolate the faults in the system.

Now, by utilizing the above FI logic and for the rest of this section, we present systematic procedures to design each residual generator in the residual bank, $r_{k}(t)$, with minimal order. For this, let us rearrange and partition the fault vector, $f(t)$, and the fault identity matrix, $D$, as follows:

$$
\begin{aligned}
f(t) & =\left[\begin{array}{l}
f_{k}(t) \\
f_{R}(t)
\end{array}\right], \\
D & =\left[\begin{array}{ll}
D_{k} & D_{R}
\end{array}\right],
\end{aligned}
$$

where $f_{k}(t)$ is the $k$ th fault, $f_{R}(t)$ contains the remaining $(l-1)$ faults, $D_{k} \in \mathbb{R}^{n \times 1}$, and $D_{R} \in \mathbb{R}^{n \times(l-1)}$.

Thus, system (1) can now be rewritten as

$$
\begin{aligned}
& \dot{x}(t)=A x(t)+B u(t)+D_{k} f_{k}(t)+D_{R} f_{R}(t), \\
& y(t)=C x(t) .
\end{aligned}
$$

Similar to Section 2, let us now consider a reduced-order functional observer for the purpose of fault isolation:

$$
\dot{\omega}_{k}(t)=N_{k} \omega_{k}(t)+G_{k} y(t)+H_{k} u(t),
$$

where $\omega_{k}(t) \in \mathbb{R}^{q}, 1 \leq q<n, N_{k} \in \mathbb{R}^{q \times q}, G_{k} \in \mathbb{R}^{q \times p}$, and $H_{k} \in \mathbb{R}^{q \times m}$ are observer parameters to be determined such that $\omega_{k}(t)$ is an asymptotic estimate of the linear function $L_{k} x(t)$ when $f(t)=0$ and $L_{k} \in \mathbb{R}^{q \times n}$ is a matrix to be determined for the purpose of fault isolation.

Let an error vector $e_{k}(t)$ be defined as follows:

$$
e_{k}(t)=\omega_{k}(t)-L_{k} x(t) \text {. }
$$

By taking the derivative of (46) and using (44) and (45), the following error dynamics is obtained:

$$
\begin{aligned}
\dot{e}_{k}(t)= & N_{k} e_{k}(t)+\left(N_{k} L_{k}+G_{k} C-L_{k} A\right) x(t) \\
& +\left(H_{k}-L_{k} B\right) u(t)-L_{k} D_{k} f_{k}(t) \\
& -L_{k} D_{R} f_{R}(t) .
\end{aligned}
$$

Proposition 9. Under no-fault conditions, $\omega_{k}(t)$ is an asymptotic estimate of the function $L_{k} x(t)$ (i.e., $e_{k}(t) \rightarrow 0$ asymptotically) for any $x(0)$ and $\omega_{k}(0)$ if

$$
\begin{gathered}
N_{k} \text { is Hurwitz, } \\
N_{k} L_{k}+G_{k} C-L_{k} A=0, \\
H_{k}-L_{k} B=0 .
\end{gathered}
$$

Proof. The proof of Proposition 9 is the same as the proof of Proposition 1; thus it is omitted here.

By using the estimate $\omega_{k}(t)$ and the system outputs, $y(t)$, the residual generator $r_{k}(t)$, which is insensitive to fault $f_{k}(t)$ and sensitive to the other faults, $f_{R}(t)$, is now proposed:

$$
r_{k}(t)=T_{k} \omega_{k}(t)+F_{k} y(t),
$$

where $r_{k}(t) \in \mathbb{R}, T_{k} \in \mathbb{R}^{1 \times q}$, and $F_{k} \in \mathbb{R}^{1 \times p}$.

Since faults $f(t)$ occur independently, matrices $T_{k}$ and $F_{k}$ need to be found to satisfy the following functions of $r_{k}(t)$ :

$$
\lim _{t \rightarrow \infty} r_{k}(t)= \begin{cases}0 & \text { if } f_{R}(t)=0 \\ c_{k} \text { or undefined } & \text { if } f_{R}(t) \neq 0,\end{cases}
$$

where $c_{k} \neq 0, f_{R}(t)=0$ means that, except fault $f_{k}(t)$ which may or may not happen, other faults do not happen, and $f_{R}(t) \neq 0$ means that any of the $(l-1)$ faults can happen in the system.

If all the conditions in Proposition 9 are satisfied, from (45), (46), and (51), the residual $r_{k}(t)$ can be governed in the following equations:

$$
\begin{aligned}
\dot{e}_{k}(t) & =N_{k} e_{k}(t)-L_{k} D_{k} f_{k}(t)-L_{k} D_{R} f_{R}(t), \\
r_{k}(t) & =T_{k} e_{k}(t)+\left(T_{k} L_{k}+F_{k} C\right) x(t) .
\end{aligned}
$$

Proposition 10. The residual generator $r_{k}(t)$ is insensitive to fault $f_{k}(t)$ and sensitive to faults $f_{R}(t)$ for any $x(0)$ and $\omega_{k}(0)$ if

$$
\begin{aligned}
T_{k} L_{k}+F_{k} C & =0, \\
L_{k} D_{k} & =0, \\
L_{k} D_{R} & \neq 0 .
\end{aligned}
$$

Proof. The proof can be constructed by following similar lines as in the proof of Proposition 3 and thus it is omitted here.

From Propositions 9 and 10, we can now determine the unknown parameters $L_{k}, N_{k}, G_{k}, H_{k}, T_{k}$, and $F_{k}$ to complete the design of the residual generator $r_{k}(t)$. Clearly, matrix $H_{k}$ can be calculated from (50) and condition (56) can be easily verified if $L_{k}$ is found. Therefore, the residual generator design now reduces to find the solutions to unknown matrices $L_{k}, N_{k}, G_{k}, T_{k}$, and $F_{k}$ which satisfy three conditions (49), (54), and (55). Here, we again assign stable eigenvalues to $N_{k}$ 
and then the fault isolation scheme can be achieved in the following sections.

As in Section 3, let us use matrix $P$ defined in (14) and the partitions in (15) and (17) and the following partitions:

$$
\begin{aligned}
L_{k} P & =\left[\begin{array}{ll}
L_{k 1} & L_{k 2}
\end{array}\right], \\
P^{-1} D_{k} & =\left[\begin{array}{l}
D_{k 1} \\
D_{k 2}
\end{array}\right],
\end{aligned}
$$

where $L_{k 1} \in \mathbb{R}^{q \times p}, L_{k 2} \in \mathbb{R}^{q \times(n-p)}, D_{k 1} \in \mathbb{R}^{p \times 1}$, and $D_{k 2} \in$ $\mathbb{R}^{(n-p) \times 1}$.

Accordingly, matrix equations (49), (54), and (55) can be partitioned to the following:

$$
\begin{aligned}
& G_{k}=L_{k 1} A_{11}+L_{k 2} A_{21}-N_{k} L_{k 1}, \\
& F_{k}=-T_{k} L_{k 1}, \\
& N_{k} L_{k 2}-L_{k 2} A_{22}-L_{k 1} A_{12}=0 \\
& T_{k} L_{k 2}=0 \\
& L_{k 1} D_{k 1}+L_{k 2} D_{k 2}=0 .
\end{aligned}
$$

Clearly, with prescribed eigenvalues for $N_{k}$ in (48), from three matrix equations (61)-(63) matrices $L_{k 1}, L_{k 2}$, and $T_{k}$ can be found. Moreover, based on (59) and (60), $G_{k}$ and $F_{k}$ are calculated, respectively, to complete the design of the residual generator. For this, we will consider two cases, namely, Cases 3 and 4 . In Case 3, we present a solution to matrix equations (61)-(63) with only a first-order residual generator designed. Indeed, it is possible that a first-order residual generator exists when $\mathscr{N}\left(\left[\begin{array}{ll}A_{12} & D_{k 1}\end{array}\right]\right) \neq 0$. This is discussed in Case 3. When a first-order residual generator does not exist, then in Case 4, based on a parametric solution approach, a residual generator can be constructed with the order as low as $[(n-p+1) /(p-1)]$.

Case 3 (first-order residual generators). In this case, we design a bank of first-order residual generators $r_{k}(t), k=$ $1,2, \ldots, l$, to isolate faults in the system. The existence conditions of each residual generator are presented in the following theorem.

Theorem 11. A first-order residual generator $r_{k}(t)$ exists if $\mathcal{N}\left(\left[\begin{array}{ll}A_{12} & D_{k 1}\end{array}\right]\right) \neq 0$ and condition (56) holds.

Proof. The proof of Theorem 11 is similar to that of Theorem 4. For completeness, it is given here. To design a first-order residual generator $r_{k}(t)$, that is, $q=1, N_{k} \in \mathbb{R}^{1 \times 1}$ can be assigned as any negative scalar, say, $N_{k}=s_{k}<0$. By letting $L_{k 2}=0$, we can arbitrarily choose $T_{k} \neq 0$ as any real number $\alpha_{k}, \alpha_{k} \neq 0$. Accordingly, (61)-(63) are now reduced to

$$
L_{k 1} \Theta_{k}=0, \quad \text { where } \Theta_{k}=\left[\begin{array}{ll}
A_{12} & D_{k 1}
\end{array}\right]
$$

As in Case 1 (see also Remark 8), a solution to (64) where $L_{k 1} \neq 0$ always exists if $\mathcal{N}\left(\Theta_{k}\right) \neq 0$ such that $\mathcal{N}\left(\Theta_{k}\right) \Theta_{k}=0$.
Therefore, solutions to $L_{k 1}$ according to (64) can be computed by first finding $\widehat{L}_{k 1}$, where

$$
\widehat{L}_{k 1}=\mathcal{N}\left(\Theta_{k}\right)
$$

and then $L_{k 1}$ is selected as any row of $\widehat{L}_{k 1}$.

Finally, if condition (56) is satisfied, that is, $L_{k} D_{R} \neq 0$, the rest of the parameters of the residual generator can be determined. This completes the proof of Theorem 11.

Remark 12. Note that whenever $p>(n+1) / 2, \mathcal{N}\left(\Theta_{k}\right) \neq$ 0 always exists since $\Theta_{k}$ is a column matrix. As a result, a solution to $L_{k 1} \neq 0$ always exists and a first-order residual generator can exist to isolate faults in the system.

Case 4 (minimal-order residual generators). In the case that a bank of first-order residual generators is not possible to design by applying the method presented in Case 3, we can employ a parametric approach to design a bank of minimalorder residual generators to isolate faults in the system. For this, as in Case 2, we assume that the pair $(C, A)$ is observable. In the remainder of this section, we present a systematic procedure which helps to design each residual generator, $r_{k}(t)$, of the residual bank. For this, we consider the case where $\operatorname{rank}\left(\left[\begin{array}{ll}A_{12} & D_{k 1}\end{array}\right]\right)=p$ and let us use similar notations as in Case 2 (Section 3 ) to state the solution of generalized Sylvester equation (61), where

$$
\begin{aligned}
N_{k} & =Q_{k}^{-1} \Lambda_{k} Q_{k}, \\
L_{k 1} & =Q_{k}\left[\begin{array}{llll}
U\left(s_{k 1}\right) b_{k 1} & \cdots & U\left(s_{k q}\right) b_{k q}
\end{array}\right]^{\top}, \\
L_{k 2} & =Q_{k}\left[\begin{array}{llll}
Z\left(s_{k 1}\right) b_{k 1} & \cdots & Z\left(s_{k q}\right) b_{k q}
\end{array}\right]^{\top} .
\end{aligned}
$$

The following theorem presents the existence condition and the minimal order of the residual generator $r_{k}(t)$.

Theorem 13. A residual generator $r_{k}(t)$, with an order as low as $[(n-p+1) /(p-1)]$, exists if condition (56) holds.

Proof. The design of residual generator $r_{k}(t)$ is reduced to solving three matrix equations (61)-(63) and with the satisfaction of condition (56). Since $Q_{k}$ is any invertible matrix, by substituting $L_{k 1}$ and $L_{k 2}$ from (67) and (68) into the transpose of (63), the following equation is obtained:

$$
\begin{aligned}
& {\left[\begin{array}{c}
D_{k 1} \\
D_{k 2}
\end{array}\right]^{\top}\left[\begin{array}{llll}
U\left(s_{k 1}\right) b_{k 1} & U\left(s_{k 2}\right) b_{k 2} & \cdots & U\left(s_{k q}\right) b_{k q} \\
Z\left(s_{k 1}\right) b_{k 1} & Z\left(s_{k 2}\right) b_{k 2} & \cdots & Z\left(s_{k q}\right) b_{k q}
\end{array}\right]} \\
& \quad=0 .
\end{aligned}
$$

From (69), the following $q$ equations are obtained:

$$
\left[\begin{array}{ll}
D_{k 1}^{\top} & D_{k 2}^{\top}
\end{array}\right]\left[\begin{array}{l}
U\left(s_{k i}\right) \\
Z\left(s_{k i}\right)
\end{array}\right] b_{k i}=0, \quad i=1,2, \ldots, q .
$$


It is a fact that there always exist nonsingular matrices $\Omega_{k i} \in \mathbb{R}^{p \times p}(i=1,2, \ldots, q), \Omega_{k i} \Omega_{k i}^{-1}=I_{p}$, such that the following partitions are satisfied:

$$
\begin{aligned}
{\left[\begin{array}{ll}
D_{k 1}^{\top} & D_{k 2}^{\top}
\end{array}\right]\left[\begin{array}{l}
U\left(s_{k i}\right) \\
Z\left(s_{k i}\right)
\end{array}\right] \Omega_{k i}=\left[\begin{array}{ll}
X_{k i 1} & X_{k i 2}
\end{array}\right], } \\
\Omega_{k i}^{-1} b_{k i}=\left[\begin{array}{l}
b_{k i 1} \\
b_{k i 2}
\end{array}\right],
\end{aligned}
$$

where $X_{k i 1} \in \mathbb{R}^{1 \times 1}, X_{k i 1} \neq 0, X_{k i 2} \in \mathbb{R}^{1 \times(p-1)}, b_{k i 1} \in \mathbb{R}^{1 \times 1}$, and $b_{k i 2} \in \mathbb{R}^{(p-1) \times 1}$.

From (70) and (71), the following equations are obtained:

$$
b_{k i}=\Omega_{k i}\left[\begin{array}{c}
-X_{k i 1}^{-1} X_{k i 2} \\
I_{(p-1)}
\end{array}\right] b_{k i 2}, \quad i=1,2, \ldots, q .
$$

Let us denote that

$$
T_{k} Q_{k}=\left[\begin{array}{llll}
t_{k 1} & t_{k 2} & \cdots & t_{k q}
\end{array}\right],
$$

where $t_{k i} \neq 0, i=1,2, \ldots, q$, are arbitrarily chosen scalars.

By substituting $L_{k 2}$ from (68) and $T_{k} Q_{k}$ from (73) into the transpose of (62), we obtain

$$
\left[\begin{array}{lllll}
Z\left(s_{k 1}\right) t_{k 1} & Z\left(s_{k 2}\right) t_{k 2} & \cdots & Z\left(s_{k q}\right) t_{k q}
\end{array}\right]\left[\begin{array}{c}
b_{k 1} \\
b_{k 2} \\
\vdots \\
b_{k q}
\end{array}\right]=0 .
$$

Substituting (72) into (74), the following equation is obtained:

$$
\Phi_{k} \widetilde{b}_{k}=0
$$

where $\Phi_{k} \in \mathbb{R}^{(n-p) \times q(p-1)}$ and $\widetilde{b}_{k} \in \mathbb{R}^{q(p-1) \times 1}$ and

$$
\begin{aligned}
\tilde{b}_{k} & =\left[\begin{array}{llll}
b_{k 12}^{\top} & b_{k 22}^{\top} & \cdots & b_{k q 2}^{\top}
\end{array}\right]^{\top}, \\
\Phi_{k i} & =t_{k i} Z\left(s_{k i}\right) \Omega_{k i}\left[\begin{array}{c}
-X_{k i 1}^{-1} X_{k i 2} \\
I_{(p-1)}
\end{array}\right], \\
\Phi_{k} & =\left[\begin{array}{llll}
\Phi_{k 1} & \Phi_{k 2} & \cdots & \Phi_{k q}
\end{array}\right] .
\end{aligned}
$$

As in (38), if we define $\mathcal{N}\left(\Phi_{k}\right)$ such that $\Phi_{k} \mathcal{N}\left(\Phi_{k}\right)=0$, then a solution to $\widetilde{b}_{k} \neq 0$ exists if $\mathcal{N}\left(\Phi_{k}\right) \neq 0$. Specifically, $\mathcal{N}\left(\Phi_{k}\right) \neq 0$ always exists if the order $q$ satisfies the following condition:

$$
q \geq\left[\frac{n-p+1}{p-1}\right],
$$

and then $\widetilde{b}_{k}$ can be taken to be any column of matrix $\widehat{b}_{k}$, where $\widehat{b}_{k}$ is computed as

$$
\widehat{b}_{k}=\mathcal{N}\left(\Phi_{k}\right) .
$$

It is noted that if we assign an order $q$ such that condition (77) holds, a solution to $\widetilde{b}_{k} \neq 0$ always exists. Therefore, with an attempt to minimize the residual generator order, we only need to prescribe the order as low as $q=[(n-p+1) /(p-1)]$ which is identified to be the lowest order satisfying (77).
Consequently, with prescribed eigenvalues of $N_{k}$ and arbitrarily chosen $t_{k i} \neq 0$, matrices $L_{k 1}, L_{k 2}$, and $L_{k}$ are then easily calculated from (67), (68), and (57), respectively. If condition (56) is satisfied, a minimal-order residual generator exists to isolate the faults. The remaining parameters $G_{k}$, $F_{k}$, and $T_{k}$ can be obtained based on (59), (60), and (73), respectively, to complete the design of residual generator $r_{k}(t)$. This completes the proof of Theorem 13.

\section{Numerical Examples}

5.1. Example 1. In this example, we consider timely detection of faults in a dynamical system which has $n=8, p=5, m=$ 2 , and $l=2$. Since we have the case where $p>(n+1) / 2$ and as discussed in Case 1 (Section 3) and Case 3 (Section 4), we can indeed design only first-order residual generators to effectively detect and isolate the faults in the system. For this example, the system matrices $C=\left[\begin{array}{ll}I_{5} & 0\end{array}\right], A, B$, and $D$ are as given below:

$$
A=\left[\begin{array}{cccccccc}
-1 & 0 & 1 & 1 & -2 & 1 & -3 & 0 \\
0 & -5 & 3 & -4 & 0 & 1 & 0 & -1 \\
1 & 1 & -8 & 3 & 0 & 2 & 1 & -2 \\
-4 & 0 & 2 & -6 & 1 & -5 & -2 & 1 \\
1 & 0 & 0 & 1 & -1 & 2 & 0 & 2 \\
-2 & 0 & 1 & 2 & 0 & -3 & -1 & 1 \\
2 & 1 & -1 & 0 & -1 & 0 & -8 & 3 \\
-2 & 0 & -1 & -4 & 0 & -6 & -3 & -2
\end{array}\right],
$$$$
B=\left[\begin{array}{cc}
1 & 0 \\
2 & 1 \\
1 & -1 \\
0 & 1 \\
3 & 1 \\
1 & -3 \\
-1 & 0 \\
1 & 1
\end{array}\right]
$$

$$
D=\left[\begin{array}{cc}
4 & 2 \\
-2.5 & -1.5 \\
2 & 3 \\
-1 & -1 \\
1 & 0 \\
-1 & 2 \\
-1 & 0 \\
4 & 2.5
\end{array}\right]
$$


Since $C$ is already in the desired form, that is, $C=\left[\begin{array}{ll}I_{5} & 0\end{array}\right]$, $P$ is an identity matrix; that is, $P=I_{8}$. Accordingly, by (17), submatrices $A_{11}, A_{12}, A_{21}$, and $A_{22}$ are obtained, where

$$
\begin{aligned}
& {\left[\begin{array}{c|cc}
A_{11} & A_{12} \\
\hline A_{21} & A_{22}
\end{array}\right]} \\
& =\left[\begin{array}{ccccc|ccc}
-1 & 0 & 1 & 1 & -2 & 1 & -3 & 0 \\
0 & -5 & 3 & -4 & 0 & 1 & 0 & -1 \\
1 & 1 & -8 & 3 & 0 & 2 & 1 & -2 \\
-4 & 0 & 2 & -6 & 1 & -5 & -2 & 1 \\
1 & 0 & 0 & 1 & -1 & 2 & 0 & 2 \\
\hline-2 & 0 & 1 & 2 & 0 & -3 & -1 & 1 \\
2 & 1 & -1 & 0 & -1 & 0 & -8 & 3 \\
-2 & 0 & -1 & -4 & 0 & -6 & -3 & -2
\end{array}\right] .
\end{aligned}
$$

Now, the design of first-order residual generators can be readily carried out to detect and isolate the faults in the system.

5.1.1. First-Order Residual Generator Detects Faults in the System. It is clear that $A_{12}$ is a column matrix and thus its null-space exists; that is, $\mathcal{N}\left(A_{12}\right) \neq 0$. As discussed in Case 1 (Section 3), a first-order functional observer exists for the FD purpose. Let us assign $N=-8 ; L_{1}$ is then computed according to (25), where

$$
L_{1}=\left[\begin{array}{lllll}
4 & -27 & 14 & 1 & 0
\end{array}\right] .
$$

Since letting $L_{2}=\left[\begin{array}{lll}0 & 0 & 0\end{array}\right]$ and by (16), matrix $L$ is obtained as

$$
L=\left[\begin{array}{llllllll}
4 & -27 & 14 & 1 & 0 & 0 & 0 & 0
\end{array}\right]
$$

By that, condition (13) is found to be satisfied since $L D=$ $\left[\begin{array}{ll}110.5 & 89.5\end{array}\right] \neq 0$. Hence, a first-order residual generator now can be designed to detect faults in the system. We complete the design by first choosing $T=0.2$ and matrices $H, G$, and $F$ are then obtained based on (9), (19), and (20), respectively, where

$$
\begin{aligned}
H & =\left[\begin{array}{lll}
-36 & -40
\end{array}\right], \\
G & =\left[\begin{array}{lllll}
38 & -67 & -75 & 156 & -7
\end{array}\right], \\
F & =\left[\begin{array}{lllll}
-0.8 & 5.4 & -2.8 & -0.2 & 0
\end{array}\right] .
\end{aligned}
$$

Figure 2 shows that the first-order residual generator can detect faults $f_{1}(t)$ and $f_{2}(t)$ in the system. Fault $f_{1}(t)$ appears at the time $t=10 \mathrm{~s}$ and clears from the time $t=20 \mathrm{~s}$. Fault $f_{2}(t)$ happens from the time $t=30 \mathrm{~s}$ to the time $t=40 \mathrm{~s}$. It is clear from the figure that while the faults are happening, the residual generator triggers them, whereas when the faults clear, the residual generator converges to zero as expected. Note also that the residual generator is insensitive to the inputs $u(t)$. It is clear from this example that the residual generator is designed by using a significantly lower-order (only first-order) functional observer. In contrast, existing

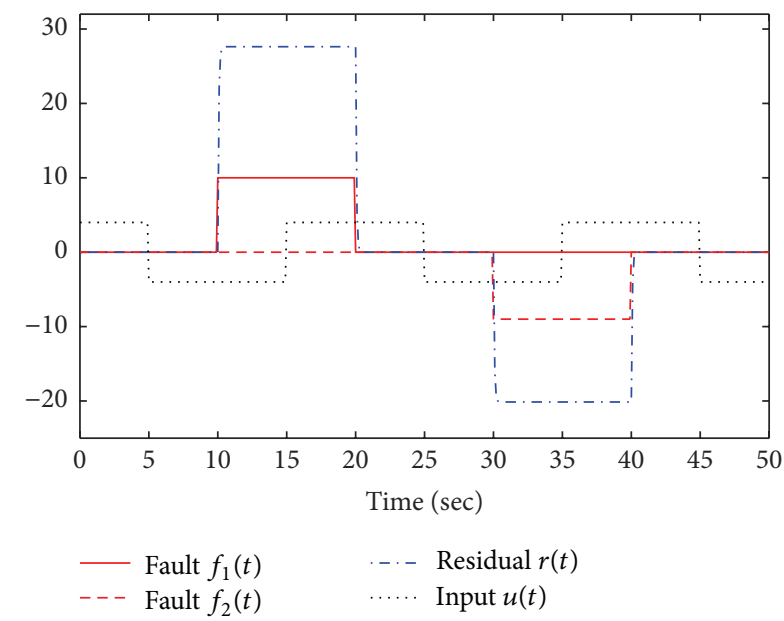

FIGURE 2: First-order observer-based residual generator detects faults in the system.

FD schemes using full-order or reduced-order state observers would give higher-order schemes. This example thus serves to highlight the attractiveness of our FD scheme proposed in this paper.

5.1.2. First-Order Residual Bank Isolates Faults in the System. In this section, we demonstrate our solution approach to isolate the faults in the system as presented in Case 3 (Section 4). For this system, there are two possible faults; thus we design a bank of two first-order residual generators, $r_{1}(t)$ and $r_{2}(t)$, to isolate the faults. Let us assign the eigenvalue for both residuals as $N_{k}=-6, k=1,2$. Now the conditions, which ensure the existence of each residual generator, will be verified and the design of the residual bank will be carried out.

Residual Generator $r_{1}(t)$. Here, $r_{1}(t)$ is designed to be insensitive to fault $f_{1}(t)$ but sensitive to $f_{2}(t)$. From partitions (43) and (58), $D_{1}, D_{R}$, and $D_{11}$ are as given below:

$$
\begin{aligned}
D_{1} & =\left[\begin{array}{llllllll}
4 & -2.5 & 2 & -1 & 1 & -1 & -1 & 4
\end{array}\right]^{\top}, \\
D_{R} & =\left[\begin{array}{llllllll}
2 & -1.5 & 3 & -1 & 0 & 2 & 0 & 2.5
\end{array}\right]^{\top}, \\
D_{11} & =\left[\begin{array}{lllll}
4 & -2.5 & 2 & -1 & 1
\end{array}\right]^{\top} .
\end{aligned}
$$

According to (65), $L_{11}$ is found by computing $\mathcal{N}\left(\Theta_{1}\right)$. This gives

$$
L_{11}=\left[\begin{array}{lllll}
-0.2353 & 0.5882 & 1.1765 & 0.9412 & 1
\end{array}\right] .
$$

Since $L_{12}=\left[\begin{array}{lll}0 & 0 & 0\end{array}\right]$, from (57), $L_{1}=\left[\begin{array}{llll}L_{11} & 0 & 0 & 0\end{array}\right]$ and condition (56) is satisfied since $L_{1} D_{R}=1.2353 \neq 0$. Hence, based on Theorem 11, first-order residual generator $r_{1}(t)$ exists for FI. Now, by assigning $T_{1}=8$, matrices $H_{1}, G_{1}$, and $F_{1}$ are calculated based on (50), (59), and (60), 


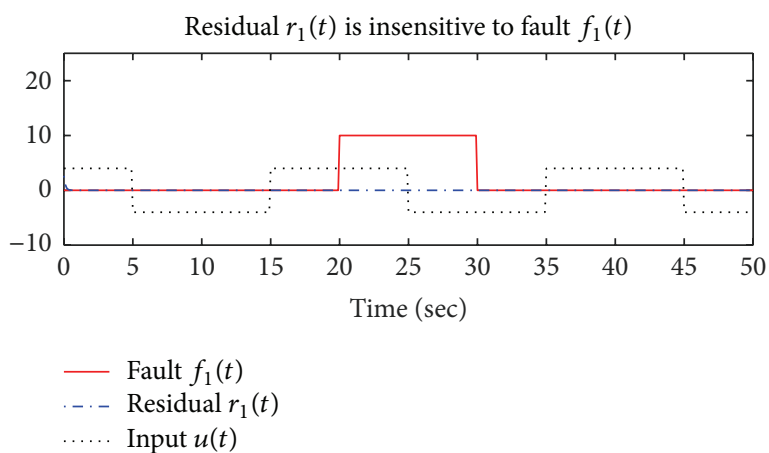

(a)

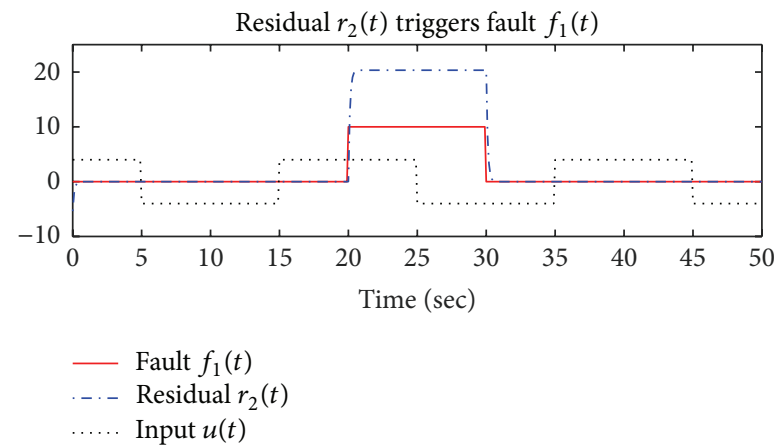

(b)

FIGURE 3: First-order observer-based residual bank isolates faults in the system.

respectively, to complete the design of residual generator $r_{1}(t)$, where

$$
\begin{aligned}
H_{1} & =\left[\begin{array}{llll}
5.1176 & 1.3529
\end{array}\right], \\
F_{1} & =\left[\begin{array}{lllll}
1.8824 & -4.7059 & -9.4118 & -7.5294 & -8
\end{array}\right], \\
G_{1} & =\left[\begin{array}{lllll}
-2.7647 & 1.7647 & 1.0588 & 1.9412 & 6.4118
\end{array}\right] .
\end{aligned}
$$

Residual Generator $r_{2}(t)$. By applying the same process as for the residual generator $r_{1}(t)$, we have the following results for the residual generator $r_{2}(t)$, which is insensitive to fault $f_{2}(t)$ and sensitive to fault $f_{1}(t)$ :

$$
\begin{aligned}
D_{2} & =\left[\begin{array}{llllllll}
2 & -1.5 & 3 & -1 & 0 & 2 & 0 & 2.5
\end{array}\right]^{\top}, \\
D_{R} & =\left[\begin{array}{llllllll}
4 & -2.5 & 2 & -1 & 1 & -1 & -1 & 4
\end{array}\right]^{\top}, \\
D_{21} & =\left[\begin{array}{lllll}
2 & -1.5 & 3 & -1 & 0
\end{array}\right]^{\top}, \\
L_{21} & =\left[\begin{array}{llllll}
-0.2905 & 0.9609 & 0.9832 & 0.9274 & 1
\end{array}\right], \\
L_{2} & =\left[\begin{array}{llll}
L_{21} & 0 & 0 & 0
\end{array}\right] .
\end{aligned}
$$

Since $L_{2} D_{R}=-1.5251 \neq 0$, condition (56) holds. Consequently, the residual generator $r_{2}(t)$ exists with a first order. Now, we can complete the design of $r_{2}(t)$ by calculating matrices $\mathrm{H}_{2}, G_{2}$, and $F_{2}$, where

$$
\begin{aligned}
H_{2} & =\left[\begin{array}{llll}
5.6145 & 1.9050
\end{array}\right], \\
F_{2} & =\left[\begin{array}{lllll}
2.3240 & -7.6872 & -7.8659 & -7.4190 & -8
\end{array}\right] \\
G_{2} & =\left[\begin{array}{lllll}
-3.1788 & 1.9441 & 2.4804 & -0.1844 & 6.5084
\end{array}\right] .
\end{aligned}
$$

Figure 3 shows that a bank of two first-order residual generators can effectively and timely isolate the faults in the system. Here, fault $f_{1}(t)$ happens in the system from the time $20 \mathrm{~s}$ and clears from the time $30 \mathrm{~s}$. By that, the output of residual generator $r_{1}(t)$ remains as zero. Meanwhile, that of the residual generator $r_{2}(t)$ derives from zero. Consequently, by observing the residual outputs, we can identify that fault $f_{1}(t)$ happened in the system. Clearly, the residual bank can effectively and timely isolate the faults in the system. This example again serves to illustrate the simplicity of our proposed FI scheme using minimal-order functional observers.
5.2. Example 2. This example is given to demonstrate Case 2 (Section 3 ) and Case 4 (Section 4 ) by considering a system with $n=8, p=3, m=2$, and $l=3$, where $C=\left[\begin{array}{ll}I_{3} & 0\end{array}\right]$, and $A, B$, and $D$ are as given below:

$$
\begin{aligned}
A & =\left[\begin{array}{l|lc}
A_{11} & A_{12} \\
\hline A_{21} & A_{22}
\end{array}\right] \\
& =\left[\begin{array}{ccc|ccccc}
-5 & 2 & 0 & 1 & 0 & 1 & 9 & -1 \\
1 & -1 & 0 & 0 & 1 & -1 & 0 & 1 \\
1 & 0 & -5 & 3 & 4 & 0 & -3 & 2 \\
\hline-3 & 1 & 1 & -8 & 3 & 1 & 4 & 2 \\
-2 & -4 & 0 & 0 & -6 & 0 & 1 & 0 \\
0 & 0 & -1 & 1 & -2 & -3 & 3 & -1 \\
2 & 0 & 2 & 3 & 7 & 1 & -7 & 0 \\
1 & 1 & 0 & -2 & 1 & 0 & 0 & -1
\end{array}\right],
\end{aligned}
$$

$$
B=\left[\begin{array}{cc}
1 & 0 \\
0 & 1 \\
1 & -1 \\
0 & 0 \\
0 & 1 \\
1 & -2 \\
1 & 1 \\
-1 & 0
\end{array}\right],
$$

$$
D=\left[\begin{array}{ccc}
1 & -2 & 1 \\
-2 & -1 & -2 \\
-2 & 1 & 3 \\
0 & -3 & 1 \\
2 & 0 & -1 \\
2 & 1 & 0 \\
-2 & -1 & 1 \\
1 & -1 & 2
\end{array}\right] \text {. }
$$


From (30)-(34), we obtain the following pair of coprime polynomial matrices $U(s)$ and $Z(s)$ :

$$
\begin{aligned}
& U(s) \\
& =\left(s^{5}+25 s^{4}+214 s^{3}+762 s^{2}+1118 s+582\right) I_{3}, \\
& Z(s)=\left(\Upsilon_{1} s^{4}+\Upsilon_{2} s^{3}+\Upsilon_{3} s^{2}+\Upsilon_{4} s+\Upsilon_{5}\right) A_{12}^{\top},
\end{aligned}
$$

where

$$
\begin{aligned}
& \Upsilon_{1}=I_{5} \text {, } \\
& \Upsilon_{2}=\left[\begin{array}{ccccc}
17 & 0 & 1 & 3 & -2 \\
3 & 19 & -2 & 7 & 1 \\
1 & 0 & 22 & 1 & 0 \\
4 & 1 & 3 & 18 & 0 \\
2 & 0 & -1 & 0 & 24
\end{array}\right], \\
& \Upsilon_{3}=\left[\begin{array}{ccccc}
87 & 3 & 25 & 31 & -32 \\
61 & 107 & -9 & 91 & 12 \\
18 & 1 & 152 & 18 & -2 \\
46 & 12 & 47 & 110 & -7 \\
31 & 0 & -19 & 5 & 186
\end{array}\right] \text {, } \\
& \Upsilon_{4}=\left[\begin{array}{ccccc}
160 & 13 & 131 & 90 & -139 \\
212 & 211 & 135 & 286 & -27 \\
79 & 12 & 378 & 87 & -33 \\
141 & 38 & 218 & 261 & -73 \\
125 & 5 & -83 & 39 & 514
\end{array}\right] \text {, } \\
& \Upsilon_{5}=\left[\begin{array}{ccccc}
90 & 12 & 150 & 72 & -168 \\
162 & 138 & 270 & 246 & -186 \\
64 & 15 & 333 & 90 & -113 \\
102 & 33 & 267 & 198 & -171 \\
116 & 9 & -33 & 54 & 359
\end{array}\right]
\end{aligned}
$$

For this example, by applying the scheme discussed in Case 2, we only need to design a residual generator based on a second-order functional observer to detect the faults in the system. Furthermore, for isolating the faults, we use only a bank of three residual generators which are designed based on third-order functional observers. The design is carried out in the following sections.

5.2.1. Second-Order Residual Generator Detects Faults in the System. As $A_{12}$ has full-row rank, that is, $\operatorname{rank}\left(A_{12}\right)=3$ and $1<p<n / 2$, this falls into Case 2 (Section 3). By that, we can design a residual generator using a functional observer which has an order of $q=[(8-3+1) / 3]=2$, that is, second order, to detect the faults in the system.
Let us assign the eigenvalues of $N$ to be $s_{1}=-4$ and $s_{2}=$ -5 and choose $Q=I_{2}$. Also, scalars $t_{i}$ are chosen as $T Q=$ $\left[\begin{array}{ll}-0.5 & -0.5\end{array}\right]$. From (38), $\mathscr{Z}$ is obtained, where

$$
\mathscr{Z}^{\top}=\left[\begin{array}{rrrrr}
-56.0 & -376 & 110.0 & -63.0 & 77.0 \\
-25.0 & -191 & 65.5 & -31.5 & 35.5 \\
-11.0 & -124 & 38.0 & -27.0 & 14.0 \\
-90.5 & -846 & 35.0 & -83.5 & 80.0 \\
-2.0 & -256 & 68.0 & -30.0 & -8.0 \\
64.5 & -194 & 17.0 & -98.5 & -80.0
\end{array}\right] \text {. }
$$

Clearly, $\mathscr{Z}$ is a row matrix; as a result, $b \neq 0$ exists and is obtained by taking any column of $\mathscr{N}(\mathscr{Z})$ in (38). Accordingly, $b_{1}$ and $b_{2}$ are obtained, where

$$
\begin{aligned}
& b_{1}=\left[\begin{array}{lll}
0.499 & -0.788 & -0.323
\end{array}\right]^{\top}, \\
& b_{2}=\left[\begin{array}{lll}
-0.046 & 0.152 & 0.014
\end{array}\right]^{\top} .
\end{aligned}
$$

From (27) and (28), $L_{1}$ and $L_{2}$ are obtained:

$$
\begin{aligned}
L_{1}= & {\left[\begin{array}{ccc}
-8.991 & 14.184 & 5.813 \\
9.521 & -31.531 & -2.842
\end{array}\right], } \\
L_{2}= & {\left[\begin{array}{cc}
9.441 & -9.441 \\
-5.465 & 5.465 \\
17.877 & -17.877 \\
-4.143 & 4.143 \\
-11.935 & 11.935
\end{array}\right]^{\top} . }
\end{aligned}
$$

Since $P=I_{8}, L=\left[\begin{array}{ll}L_{1} & L_{2}\end{array}\right]$ and it is easy to verify condition (13) that

$$
L D=\left[\begin{array}{ccc}
-27.811 & 15.241 & -33.030 \\
57.093 & 4.016 & 77.164
\end{array}\right] \neq 0 .
$$

Thus, condition (13) is satisfied and hence a residual generator using a second-order functional observer can be constructed to detect the faults. The parameters of the observer and the residual generator are obtained by (26), (19), (9), (20), and (36), where

$$
\begin{aligned}
& N=\left[\begin{array}{cc}
-4 & 0 \\
0 & -5
\end{array}\right] \text {, } \\
& G=\left[\begin{array}{ccc}
-8.628 & 43.934 & -22.533 \\
3.243 & -126.445 & 16.720
\end{array}\right] \text {, } \\
& H=\left[\begin{array}{cc}
22.491 & -36.989 \\
-18.990 & 16.672
\end{array}\right] \text {, } \\
& F=\left[\begin{array}{lll}
0.265 & -8.673 & 1.485
\end{array}\right] \text {, } \\
& T=\left[\begin{array}{ll}
-0.5 & -0.5
\end{array}\right] .
\end{aligned}
$$

Figure 4 indicates that the residual generator can detect the faults $f_{1}(t), f_{2}(t)$, and $f_{3}(t)$ in the system. It is clear in this example that the design of the residual generator is very simple and systematic. 


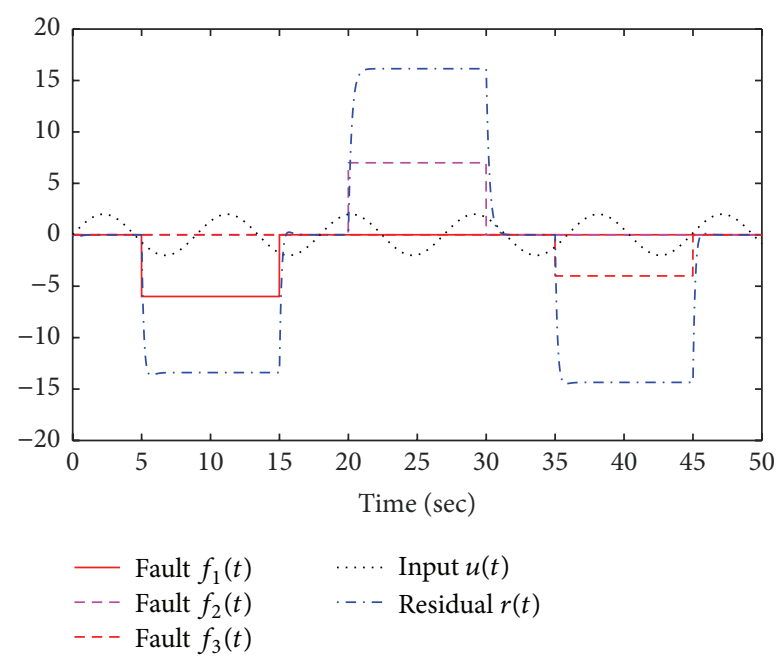

FIGURE 4: Second-order observer-based residual generator detects the faults in the system.

5.2.2. Third-Order Residual Bank Isolates the Faults in the System. By applying the scheme in Case 4, for this system, we now proceed to design a bank of three residual generators which are based on third-order functional observers, $q=$ $[(8-3+1) /(3-1)]=3$, to isolate the faults happening in the system.

To design the residual bank, we, for the sake of simplicity, firstly assign the poles of $N_{k}$ to be at $s_{k 1}=-4, s_{k 2}=-5$, and $s_{k 3}=-7, k=1,2,3 . Q_{k}$ is chosen as $Q_{k}=I_{3}$, and $T_{k} Q_{k}$ is chosen as $T_{k} Q_{k}=\left[\begin{array}{lll}1 & 1 & 1\end{array}\right]$ for all the three residual generators. In the remainder of this example, we will verify the conditions, which guarantee the existence of each residual generator, and complete the design by determining all the necessary parameters.

Residual Generator $r_{1}(t)$. This residual generator is designed such that it is insensitive to fault $f_{1}(t)$ but sensitive to faults $f_{2}(t)$ and $f_{3}(t)$. From partitions (43) and (58), $D_{1}, D_{R}, D_{11}$, and $D_{12}$ are obtained as

$$
\begin{aligned}
D_{1} & =\left[\begin{array}{llllllll}
1 & 1 & 0 & 0 & 1 & 0 & -2 & -1
\end{array}\right]^{\top}, \\
D_{R} & =\left[\begin{array}{cccccccc}
-2 & -1 & 1 & -3 & 0 & 1 & -1 & -1 \\
1 & -2 & 3 & 1 & -1 & 0 & 1 & 2
\end{array}\right]^{\top}, \\
D_{11} & =\left[\begin{array}{lll}
1 & 1 & 0
\end{array}\right]^{\top}, \\
D_{12} & =\left[\begin{array}{lllll}
0 & 1 & 0 & -2 & -1
\end{array}\right]^{\top} .
\end{aligned}
$$

From (75), $\Phi_{1}$ is obtained, where

$$
\Phi_{1}^{\top}=\left[\begin{array}{ccccc}
-4.42 & 16.64 & -24.11 & 1.78 & 3.82 \\
-7.59 & 49.36 & -17.89 & 20.72 & 12.68 \\
-19.21 & 295.01 & -127.02 & 38.58 & 36.52 \\
-106.06 & 602.41 & -42.87 & 218.16 & 139.72 \\
-44.15 & 119.43 & -15.88 & 6.03 & 29.01 \\
-174.63 & 246.42 & 19.41 & 83.22 & 93.74
\end{array}\right] \text {. }
$$

Since $\Phi_{1}$ is a row matrix, $\tilde{b}_{1} \neq 0$ in (75) exists and is taken from $\mathscr{N}\left(\Phi_{1}\right)$. Accordingly, from (72), $b_{11}, b_{12}$, and $b_{13}$ are obtained, where

$$
\begin{aligned}
& b_{11}=\left[\begin{array}{lll}
0.142 & -0.684 & 0.721
\end{array}\right]^{\top}, \\
& b_{12}=\left[\begin{array}{lll}
-0.018 & 0.057 & -0.083
\end{array}\right]^{\top}, \\
& b_{13}=\left[\begin{array}{lll}
-0.007 & 0.026 & 0.023
\end{array}\right]^{\top} .
\end{aligned}
$$

From (67) and (68), $L_{11}$ and $L_{12}$ are obtained:

$$
\begin{aligned}
L_{11} & =\left[\begin{array}{ccc}
-2.555 & 12.318 & -12.985 \\
3.685 & -11.767 & 17.173 \\
0.620 & -2.302 & -2.108
\end{array}\right], \\
L_{12} & =\left[\begin{array}{ccc}
-2.450 & 7.670 & -5.219 \\
24.219 & -33.046 & 8.827 \\
3.598 & -3.646 & 0.048 \\
13.725 & -15.829 & 2.103 \\
6.532 & -9.470 & 2.938
\end{array}\right] .
\end{aligned}
$$

By (57) and $P=I_{8}$ that $L_{1}=\left[\begin{array}{ll}L_{11} & L_{12}\end{array}\right]$ and condition (56) is satisfied since

$$
L_{1} D_{R}=\left[\begin{array}{ccc}
-29.503 & 20.212 & 9.619 \\
-66.028 & 84.683 & -7.166
\end{array}\right]^{\top} \neq 0
$$

Thus, residual generator $r_{1}(t)$ based on a third-order functional observer exists, and from (50), (59), (60), and (73), the rest of the parameters of the residual generator are obtained, where

$$
\begin{aligned}
& H_{1}=\left[\begin{array}{cc}
-4.749 & 56.053 \\
10.852 & -70.521 \\
-2.273 & 10.639
\end{array}\right], \\
& G_{1}=\left[\begin{array}{ccc}
-5.217 & -60.950 & 34.388 \\
7.361 & 90.688 & -20.341 \\
1.980 & -50.161 & -5.276
\end{array}\right], \\
& F_{1}=\left[\begin{array}{lll}
-1.751 & 1.751 & -2.079
\end{array}\right], \\
& T_{1}=\left[\begin{array}{lll}
1 & 1 & 1
\end{array}\right] .
\end{aligned}
$$


By applying the same procedure as for designing $r_{1}(t)$, the following results are obtained for residual generators $r_{2}(t)$ and $r_{3}(t)$.

Residual Generator $r_{2}(t)$. This residual generator is designed such that it is insensitive to fault $f_{2}(t)$ but sensitive to faults $f_{1}(t)$ and $f_{3}(t)$. The following results are obtained:

$$
\begin{aligned}
& D_{2}=\left[\begin{array}{llllllll}
-2 & -1 & 1 & -3 & 0 & 1 & -1 & -1
\end{array}\right]^{\top}, \\
& D_{R}=\left[\begin{array}{cccccccc}
1 & 1 & 0 & 0 & 1 & 0 & -2 & -1 \\
1 & -2 & 3 & 1 & -1 & 0 & 1 & 2
\end{array}\right]^{\top} \text {, } \\
& D_{21}=\left[\begin{array}{lll}
-2 & -1 & 1
\end{array}\right]^{\top} \text {, } \\
& D_{22}=\left[\begin{array}{lllll}
-3 & 0 & 1 & -1 & -1
\end{array}\right]^{\top} \text {, } \\
& b_{21}=\left[\begin{array}{lll}
0.311 & -0.870 & 0.451
\end{array}\right]^{\top} \text {, } \\
& b_{22}=\left[\begin{array}{lll}
-0.038 & 0.143 & -0.066
\end{array}\right]^{\top} \text {, } \\
& b_{23}=\left[\begin{array}{lll}
0.012 & -0.114 & 0.037
\end{array}\right]^{\top} \text {, } \\
& L_{21}=\left[\begin{array}{ccc}
-5.595 & 15.664 & -8.121 \\
7.945 & -29.678 & 13.729 \\
-1.099 & 10.261 & -3.347
\end{array}\right] \text {, } \\
& L_{22}=\left[\begin{array}{ccc}
1.229 & 2.172 & -3.401 \\
13.219 & -17.184 & 3.965 \\
11.325 & -14.487 & 3.161 \\
8.705 & -10.821 & 2.115 \\
1.283 & -2.166 & 0.883
\end{array}\right]^{\top}, \\
& L_{2} D_{R}=\left[\begin{array}{ccc}
12.279 & -4.025 & -4.020 \\
-62.006 & 112.689 & -35.150
\end{array}\right]^{\top} \neq 0 .
\end{aligned}
$$

Since $L_{2} D_{R} \neq 0$, condition (56) is satisfied. Hence, residual generator $r_{2}(t)$ using a third-order functional observer exists and its design is completed by obtaining the following parameters:

$$
\begin{aligned}
H_{2} & =\left[\begin{array}{cc}
5.031 & 23.0595 \\
-1.468 & -42.438 \\
-0.053 & 13.366
\end{array}\right], \\
G_{2} & =\left[\begin{array}{ccc}
1.708 & -14.560 & 15.436 \\
-11.903 & -34.079 & -4.983 \\
12.100 & 40.992 & -9.027
\end{array}\right], \\
F_{2} & =\left[\begin{array}{lll}
-1.251 & 3.752 & -2.260
\end{array}\right], \\
T_{2} & =\left[\begin{array}{lll}
1 & 1 & 1
\end{array}\right] .
\end{aligned}
$$

Residual Generator $r_{3}(t)$. This residual generator is designed such that it is insensitive to fault $f_{3}(t)$ but sensitive to faults $f_{1}(t)$ and $f_{2}(t)$. The following results are obtained:

$$
\begin{aligned}
& D_{3}=\left[\begin{array}{llllllll}
1 & -2 & 3 & 1 & -1 & 0 & 1 & 2
\end{array}\right]^{\top}, \\
& D_{R}=\left[\begin{array}{cccccccc}
1 & 1 & 0 & 0 & 1 & 0 & -2 & -1 \\
-2 & -1 & 1 & -3 & 0 & 1 & -1 & -1
\end{array}\right]^{\top}, \\
& D_{31}=\left[\begin{array}{lll}
1 & -2 & 3
\end{array}\right]^{\top}, \\
& D_{32}=\left[\begin{array}{lllll}
1 & -1 & 0 & 1 & 2
\end{array}\right]^{\top} \text {, } \\
& b_{31}=\left[\begin{array}{lll}
0.005 & -0.515 & 0.846
\end{array}\right]^{\top}, \\
& b_{32}=\left[\begin{array}{lll}
-0.003 & 0.051 & -0.107
\end{array}\right]^{\top} \text {, } \\
& b_{33}=\left[\begin{array}{lll}
-0.007 & -0.031 & 0.056
\end{array}\right]^{\top}, \\
& L_{31}=\left[\begin{array}{ccc}
-0.096 & 9.269 & -15.237 \\
0.673 & -10.609 & 22.336 \\
0.617 & 2.822 & -5.040
\end{array}\right] \text {, } \\
& L_{32}=\left[\begin{array}{ccc}
-6.524 & 13.4706 & -6.9468 \\
17.257 & -21.027 & 3.770 \\
1.943 & -3.059 & 1.116 \\
13.946 & -18.635 & 4.689 \\
12.033 & -15.847 & 3.814
\end{array}\right]^{\top} \\
& L_{3} D_{R}=\left[\begin{array}{ccc}
34.384 & -49.531 & 9.259 \\
-28.778 & 22.610 & 4.358
\end{array}\right]^{\top} \neq 0 .
\end{aligned}
$$

Since $L_{3} D_{R} \neq 0$, condition (56) is satisfied. Hence, the residual $r_{3}(t)$ exists with a third order. The rest of the parameters of the residual are obtained, where

$$
\begin{aligned}
& H_{3}=\left[\begin{array}{cc}
-11.478 & 51.822 \\
17.162 & -66.490 \\
-2.432 & 14.090
\end{array}\right], \\
& G_{3}=\left[\begin{array}{ccc}
19.109 & -35.905 & 34.661 \\
-39.747 & 40.643 & -20.740 \\
25.508 & -0.046 & -8.764
\end{array}\right], \\
& F_{3}=\left[\begin{array}{lll}
-1.194 & -1.482 & -2.059
\end{array}\right], \\
& T_{3}=\left[\begin{array}{lll}
1 & 1 & 1
\end{array}\right] .
\end{aligned}
$$




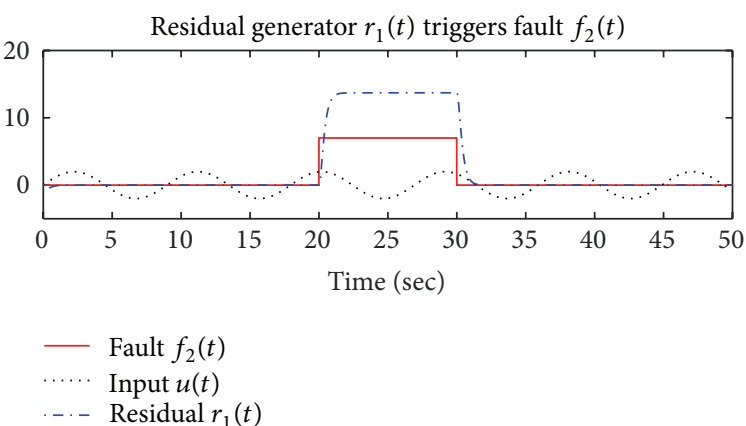

(a)

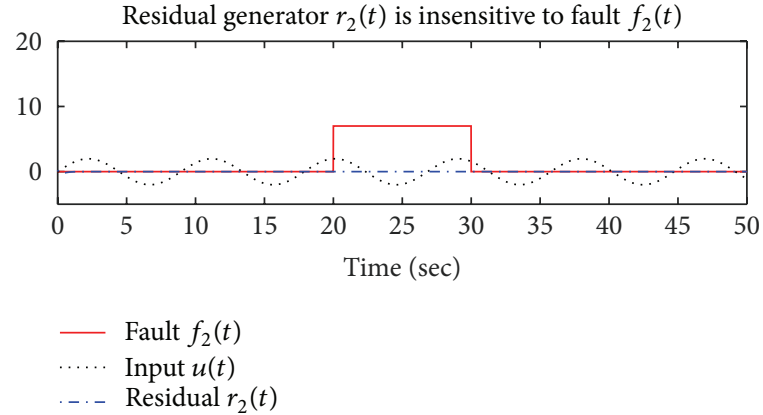

(b)

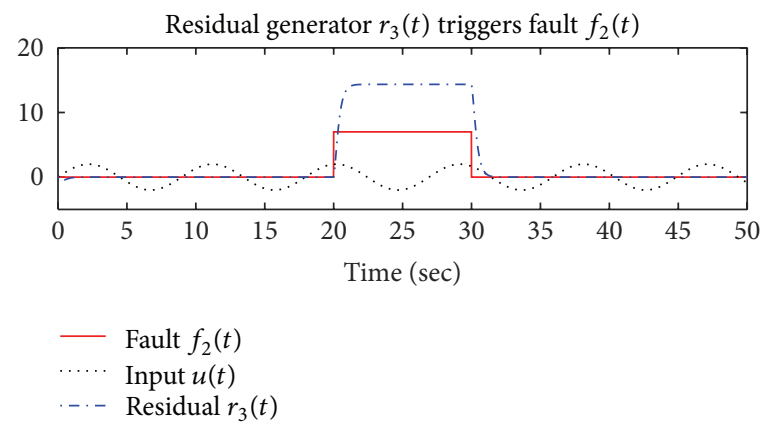

(c)

FiguRE 5: Third-order observer-based residual bank isolates the faults in the system.

Figure 5 indicates that a bank of three third-order residual generators can isolate the faults $f_{1}(t), f_{2}(t)$, and $f_{3}(t)$ in the system. In the figure, when fault $f_{2}(t)$ occurs in the system, the residual $r_{2}(t)$ is insensitive to the fault; thus its output remains as zero. Meanwhile, the residual generators $r_{1}(t)$ and $r_{3}(t)$ trigger the fault. By that we can isolate fault $f_{2}(t)$ in the system. This example again further highlights the efficiency of our proposed FI scheme in this paper.

\section{Conclusion}

This work has presented novel FD and FI schemes using minimal-order functional observers to construct residual generators to timely detect and isolate actuator faults in dynamical systems. The proposed method is based on solving a generalized Sylvester matrix equation via a parametric approach. Existence conditions and systematic procedures for designing the proposed FD and FI schemes have been presented. The reduced order and the simplicity are the hallmark of the proposed novel FD and FI schemes. It is envisaged that these proposed schemes will have widespread applications in control systems to detect and isolate actuator and/or component faults.

\section{Competing Interests}

The authors declare that they have no competing interests.

\section{References}

[1] Z. Gao, X. Liu, and M. Z. Q. Chen, "Unknown input observerbased robust fault estimation for systems corrupted by partially decoupled disturbances," IEEE Transactions on Industrial Electronics, vol. 63, no. 4, pp. 2537-2547, 2016.

[2] A. S. Willsky, "A survey of design methods for failure detection in dynamic systems," Automatica, vol. 12, no. 6, pp. 601-611, 1976.

[3] M. Hou and P. C. Muller, "Design of observers for linear systems with unknown inputs," IEEE Transactions on Automatic Control, vol. 37, no. 6, pp. 871-875, 1992.

[4] M. Aldeen and R. Sharma, "Estimation of states, faults and unknown disturbances in non-linear systems," International Journal of Control, vol. 81, no. 8, pp. 1195-1201, 2008.

[5] Q. P. Ha and H. Trinh, "State and input simultaneous estimation for a class of nonlinear systems," Automatica, vol. 40, no. 10, pp. 1779-1785, 2004.

[6] Z. Gao and H. Wang, "Descriptor observer approaches for multivariable systems with measurement noises and application in fault detection and diagnosis," Systems \& Control Letters, vol. 55 , no. 4 , pp. 304-313, 2006.

[7] H. Trinh and Q. P. Ha, "State and input simultaneous estimation for a class of time-delay systems with uncertainties," IEEE Transactions on Circuits and Systems II: Express Briefs, vol. 54, no. 6, pp. 527-531, 2007.

[8] S. S. Delshad, A. Johansson, M. Darouach, and T. Gustafsson, "Robust state estimation and unknown inputs reconstruction 
for a class of nonlinear systems: multiobjective approach," Automatica, vol. 64, pp. 1-7, 2016.

[9] J. Chen and R. J. Patton, Robust Model-Based Fault Diagnosis for Dynamic Systems, Kluwer Academic, Norwell, Mass, USA, 1999.

[10] P. M. Frank, "Fault diagnosis in dynamic systems using analytical and knowledge-based redundancy. a survey and some new results," Automatica, vol. 26, no. 3, pp. 459-474, 1990.

[11] J. Yang and F. Zhu, "FDI design for uncertain nonlinear systems with both actuator and sensor faults," Asian Journal of Control, vol. 17, no. 1, pp. 213-224, 2015.

[12] P. M. Frank and X. Ding, "Survey of robust residual generation and evaluation methods in observer-based fault detection systems," Journal of Process Control, vol. 7, no. 6, pp. 403-424, 1997.

[13] R. J. Patton and J. Chen, "Observer-based fault detection and isolation: robustness and applications," Control Engineering Practice, vol. 5, no. 5, pp. 671-682, 1997.

[14] G. R. Duan and R. J. Patton, "Robust fault detection using Luenbergertype unknown input observers-a parametric approach," International Journal of Systems Science, vol. 32, no. 4, pp. 533540, 2001

[15] J. Su, W.-H. Chen, and B. Li, "Disturbance observer based fault diagnosis," in Proceedings of the 33rd Chinese Control Conference (CCC '14), pp. 3024-3029, Nanjing, China, July 2014.

[16] G. R. Duan and R. J. Patton, "Robust fault detection in linear systems using Luenberger observers," in Proceedings of the UKACC International Conference on Control, vol. 2, pp. 14681473, Swansea, UK, September 1998.

[17] W. Ge and C. Z. Fang, "Detection of faulty components via robust observation," International Journal of Control, vol. 47, no. 2, pp. 581-599, 1988.

[18] H. Liu, D. Liu, C. Lu, and X. Wang, "Fault diagnosis of hydraulic servo system using the unscented Kalman Filter," Asian Journal of Control, vol. 16, no. 6, pp. 1713-1725, 2014.

[19] F. Zhu and F. Cen, "Full-order observer-based actuator fault detection and reduced-order observer-based fault reconstruction for a class of uncertain nonlinear systems," Journal of Process Control, vol. 20, no. 10, pp. 1141-1149, 2010.

[20] C. Edwards, S. K. Spurgeon, and R. J. Patton, "Sliding mode observers for fault detection and isolation," Automatica, vol. 36, no. 4, pp. 541-553, 2000.

[21] O. A. Z. Sotomayor and D. Odloak, "Observer-based fault diagnosis in chemical plants," Chemical Engineering Journal, vol. 112, no. 1-3, pp. 93-108, 2005.

[22] J. Chen, R. J. Patton, and H.-Y. Zhang, "Design of unknown input observers and robust fault detection filters," International Journal of Control, vol. 63, no. 1, pp. 85-105, 1996.

[23] P. Rosa and C. Silvestre, "Fault detection and isolation of LPV systems using set-valued observers: an application to a fixedwing aircraft," Control Engineering Practice, vol. 21, no. 3, pp. 242-252, 2013.

[24] T.-G. Park, "Designing fault detection observers for linear systems with mismatched unknown inputs," Journal of Process Control, vol. 23, no. 8, pp. 1185-1196, 2013.

[25] F. Xu, V. Puig, C. Ocampo-Martinez, F. Stoican, and S. Olaru, "Actuator-fault detection and isolation based on set-theoretic approaches," Journal of Process Control, vol. 24, no. 6, pp. 947956, 2014.
[26] M. Hou and P. C. Muller, "Fault detection and isolation observers," International Journal of Control, vol. 60, no. 5, pp. 827-846, 1994.

[27] J. Gertler, "Fault detection and isolation using parity relations," Control Engineering Practice, vol. 5, no. 5, pp. 653-661, 1997.

[28] D. Koenig and S. Mammar, "Design of a class of reduced order unknown inputs nonlinear observer for fault diagnosis," in Proceedings of the American Control Conference, vol. 3, pp. 21432147, Arlington, Va, USA, June 2001.

[29] A. Varga, "On designing least order residual generators for fault detection and isolation," in Proceedings of the 16th International Conference on Control Systems and Computer Science, pp. 323330, Bucharest, Romania, May 2007.

[30] Y. Xiong and M. Saif, "Unknown disturbance inputs estimation based on a state function observer design," Automatica, vol. 39, no. 8, pp. 1389-1398, 2003.

[31] Z. Yuan, G. C. Vansteenkiste, and C. Wen, "Improving the observer-based FDI design for efficient fault isolation," International Journal of Control, vol. 68, no. 1, pp. 197-218, 1997.

[32] P. S. Teh and H. Trinh, "Design of unknown input functional observers for nonlinear systems with application to fault diagnosis," Journal of Process Control, vol. 23, no. 8, pp. 1169-1184, 2013.

[33] H. Trinh, T. Fernando, K. Emami, and D. C. Huong, "Fault detection of dynamical systems using first-order functional observers," in Proceedings of the 8th IEEE International Conference on Industrial and Information Systems (ICIIS '13), pp. 197200, IEEE, Peradeniya, Sri Lanka, December 2013.

[34] D. C. Huong, H. Trinh, H. M. Tran, and T. Fernando, "Approach to fault detection of time-delay systems using functional observers," Electronics Letters, vol. 50, no. 16, pp. 1132-1134, 2014.

[35] K. Emami, T. Fernando, B. Nener, H. Trinh, and Y. Zhang, "A functional observer based fault detection technique for dynamical systems," Journal of the Franklin Institute, vol. 352, no. 5, pp. 2113-2128, 2015.

[36] M. Darouach, "Existence and design of functional observers for linear systems," IEEE Transactions on Automatic Control, vol. 45, no. 5, pp. 940-943, 2000.

[37] H. Trinh and T. Fernando, "On the existence and design of functional observers for linear systems," in Proceedings of the IEEE International Conference on Mechatronics and Automation (ICMA '07), pp. 1974-1979, Harbin, China, August 2007.

[38] H. Trinh and T. Fernando, Functional Observers for Dynamical Systems, Springer, Berlin, Germany, 2012.

[39] F. Rotella and I. Zambettakis, "On functional observers for linear time-varying systems," IEEE Transactions on Automatic Control, vol. 58, no. 5, pp. 1354-1360, 2013.

[40] T. Fernando and H. Trinh, "A system decomposition approach to the design of functional observers," International Journal of Control, vol. 87, no. 9, pp. 1846-1860, 2014.

[41] T. L. Fernando, H. M. Trinh, and L. Jennings, "Functional observability and the design of minimum order linear functional observers," IEEE Transactions on Automatic Control, vol. 55, no. 5, pp. 1268-1273, 2010.

[42] G.-R. Duan, "Solutions of the equation $A V+B W=V F$ and their application to eigenstructure assignment in linear systems," IEEE Transactions on Automatic Control, vol. 38, no. 2, pp. 276280, 1993. 
[43] J. Su and W. H. Chen, "Fault diagnosis for vehicle lateral dynamics with robust threshold," in Proceedings of the IEEE International Conference on Industrial Technology, Taipei, Taiwan, March 2016.

[44] A. Termehchy, A. Afshar, and M. Javidsharifi, "A novel design of unknown input observer for fault diagnosis in the Tennessee-Eastman process system to solve non-minimum phase problem," in Proceedings of the IEEE International Conference on Smart Instrumentation, Measurement and Applications (ICSIMA '13), pp. 1-6, Kuala Lumpur, Malaysia, November 2013. 


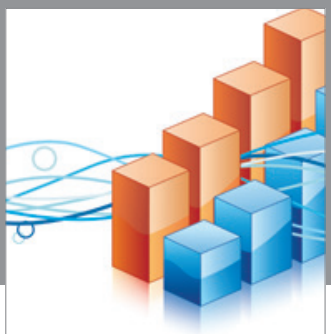

Advances in

Operations Research

vatem alat4

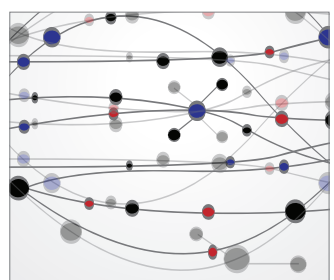

\section{The Scientific} World Journal
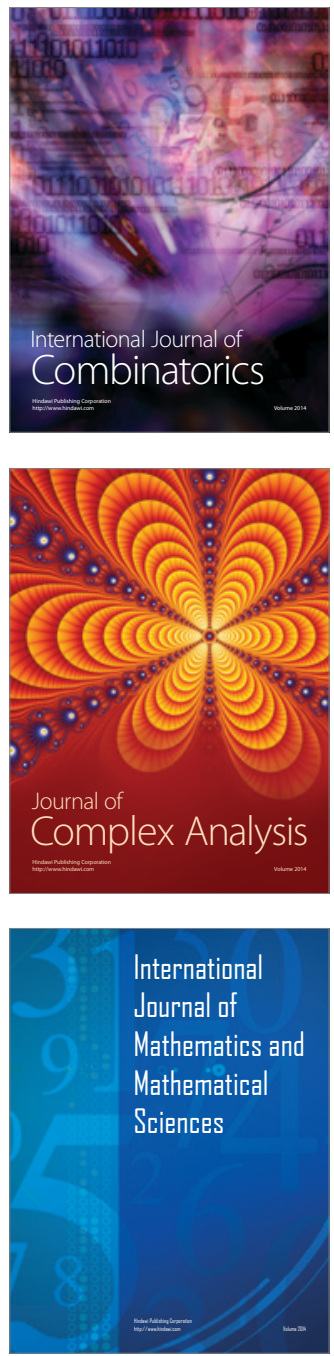
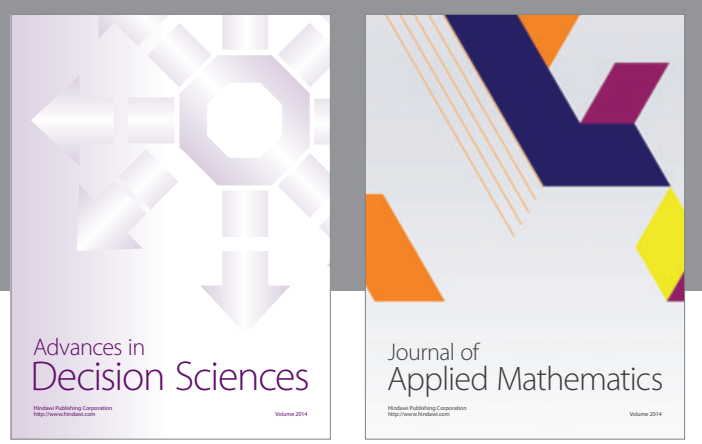

Algebra

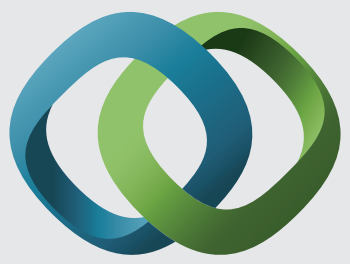

\section{Hindawi}

Submit your manuscripts at

http://www.hindawi.com
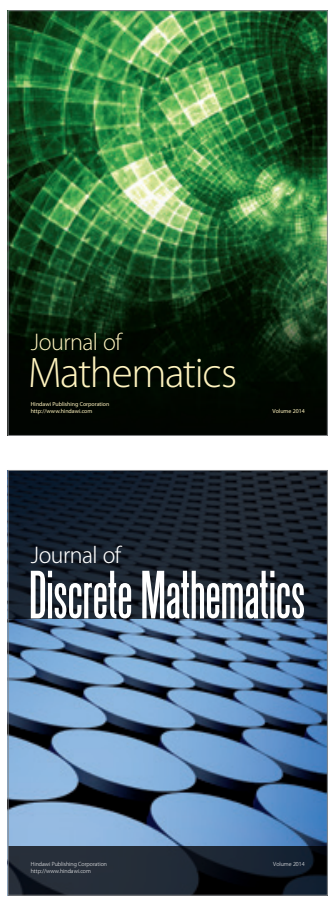

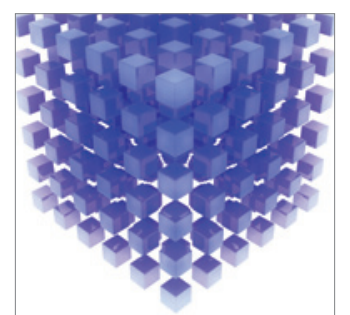

Mathematical Problems in Engineering
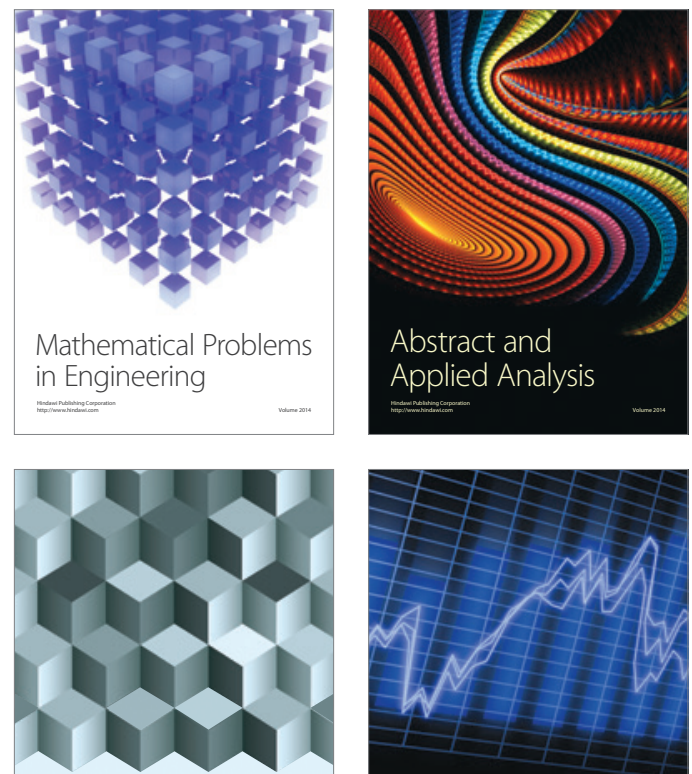

Journal of

Function Spaces

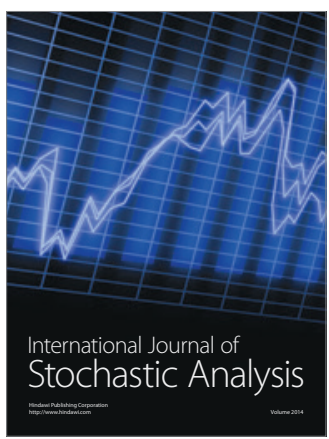

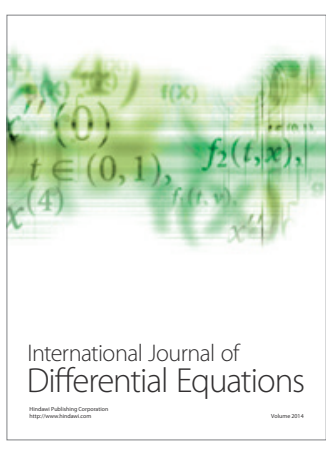
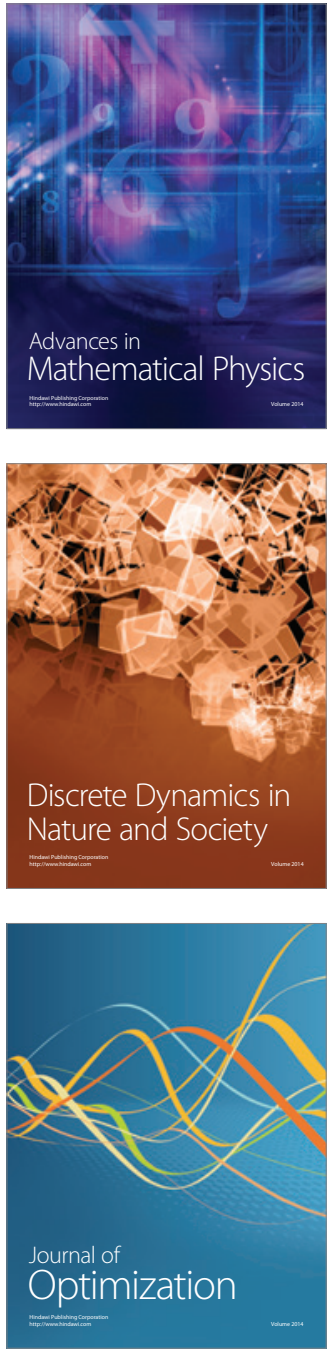